ARTICLE

\title{
The NUCKS1-SKP2-p21/p27 axis controls $S$ phase entry
}

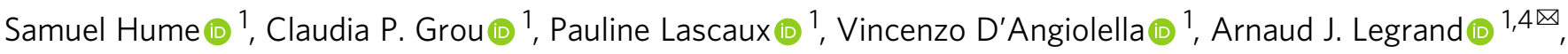 \\ Kristijan Ramadan (10 ${ }^{1 凶} \&$ Grigory L. Dianov ${ }^{1,2,3 凶}$
}

Efficient entry into $\mathrm{S}$ phase of the cell cycle is necessary for embryonic development and tissue homoeostasis. However, unscheduled S phase entry triggers DNA damage and promotes oncogenesis, underlining the requirement for strict control. Here, we identify the NUCKS1-SKP2-p21/p27 axis as a checkpoint pathway for the G1/S transition. In response to mitogenic stimulation, NUCKS1, a transcription factor, is recruited to chromatin to activate expression of SKP2, the F-box component of the SCFSKP2 ubiquitin ligase, leading to degradation of p21 and p27 and promoting progression into S phase. In contrast, DNA damage induces p53-dependent transcriptional repression of NUCKS1, leading to SKP2 downregulation, p21/p27 upregulation, and cell cycle arrest. We propose that the NUCKS1-SKP2p21/p27 axis integrates mitogenic and DNA damage signalling to control S phase entry. The Cancer Genome Atlas (TCGA) data reveal that this mechanism is hijacked in many cancers, potentially allowing cancer cells to sustain uncontrolled proliferation.

\footnotetext{
${ }^{1}$ Medical Research Council Oxford Institute for Radiation Oncology, Department of Oncology, University of Oxford, OX3 7DQ Oxford, UK. ${ }^{2}$ Institute of Cytology and Genetics, Siberian Branch of the Russian Academy of Sciences, Lavrentieva 10, 630090 Novosibirsk, Russia. ${ }^{3}$ Novosibirsk State University, Novosibirsk, Russian Federation 630090, Russia. ${ }^{4}$ Present address: Breast Cancer Now Toby Robins Research Centre, The Institute of Cancer Research,

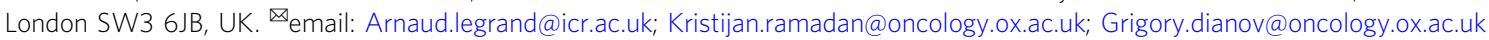


E ntry into S phase of the cell cycle is essential to sustain the proliferation that permits embryonic development and tissue repair ${ }^{1}$, but unscheduled $S$ phase entry induces replication stress, DNA damage, and oncogenesis ${ }^{2-5}$. G1/S progression must therefore be strictly controlled ${ }^{6-8}$. S phase entry is driven by mitogens, which increase the ratio of G1/S cyclins: cyclin-dependent kinase (CDK) inhibitors and activate G1/S CDKs as a result. In contrast, DNA damage inhibits $S$ phase entry, stimulating p53 signalling to reduce the G1/S cyclin: CDK inhibitor ratio and prevent G1/S CDK activity ${ }^{9}$. Only cells whose mitogenic signalling outcompetes their DNA damage load are permitted to enter $S$ phase ${ }^{6,10-14}$, which must be achieved through the integration of these antagonistic stimuli by signalling hubs. However, signalling hubs that achieve this goal are not wellcharacterised $^{6}$.

The transcription factor Nuclear Ubiquitous Casein kinase and cyclin-dependent Kinase Substrate 1 (NUCKS1) has emerged in the light of recent studies as a promising candidate for one such signalling hub. NUCKS1, a member of the high mobility group family of proteins ${ }^{15}$, increases chromatin accessibility at target promoters to enable the recruitment of RNA polymerase $\mathrm{II}^{16}$. So far, the only direct transcriptional targets identified for NUCKS1 regulate insulin receptor signalling ${ }^{16}$. However, NUCKS1 is known to affect cell cycle progression and proliferation in mammary epithelial cells ${ }^{17}$ and gastric cancer cells ${ }^{18}$, and also plays a role in the protection of replication fidelity by regulating double-strand break (DSB) repair ${ }^{19-22}$. In addition, NUCKS1 is a phosphorylation substrate for $\mathrm{CDK} 2$ and $\mathrm{CDK} 1$, the major kinases controlling the G1/S and G2/M transitions ${ }^{23-28}$, and for the DNA damage response (DDR) kinases ATM and DNA$\mathrm{PK}^{29,30}$. Furthermore, $\mathrm{Rb}-\mathrm{E} 2 \mathrm{~F}^{31}$ and $\mathrm{p} 53^{32}$ have been detected in the proximity of the NUCKS1 promoter by genome wide ChIPSeq, suggesting that NUCKS1 expression might be regulated by the cell cycle or by DNA damage.

NUCKS1 also exhibits oncogenic properties, and its overexpression, correlating with poor patient prognosis, has been reported in a number of cancers ${ }^{33-38}$. Furthermore, NUCKS1 depletion inhibits-while its overexpression promotes-xenograft tumour growth $18,39,40$, suggesting a direct role in tumourigenesis.

Altogether, these studies suggest a potentially important role for NUCKS1 in cell cycle progression. However, mechanistic details explaining how NUCKS1 does this are unknown. In particular, whether NUCKS1 employs transcriptional control of the cell cycle-and which putative targets of NUCKS1 are involvedhas not been established. The precise cell cycle phase affected by NUCKS1 is also not known, and how NUCKS1 is regulated throughout the cell cycle, by mitogens, or following DNA damage, has not been explored.

Here, we show that $\mathrm{S}$ phase Kinase-associated Protein 2 (SKP2) is a transcriptional target for NUCKS1 in late G1 phase, and identify the SKP2-p21/p27 axis as a pathway controlled by NUCKS1. SKP2 is a substrate-recruiting F-box protein, which forms, along with SKP1, CUL1, and RBX1, the SCFSKP2 ubiquitin ligase complex ${ }^{41}$. During the G1/S transition, SKP2 directs SCFSKP2 for degradation of the CDK inhibitors p21 and p27, relieving $\mathrm{p} 21 / \mathrm{p} 27-$ mediated inhibition of cyclin E-CDK2 $12,14,42,43$. In this way, SCFSKP2 controls cell cycle and cancer progression ${ }^{44-46}$.

We find that the SKP2-p21/p27 axis acts through NUCKS1 to integrate mitogenic and DNA damage signalling at the G1/S transition. We show that NUCKS1 is stimulated by mitogens to promote SKP2 expression and consequent p21/p27 degradation, enabling $S$ phase entry. In contrast, DNA damage inhibits NUCKS1 through p53, reducing SKP2 levels, increasing p21/p27 levels, and blocking $S$ phase entry. In this way, the NUCKS1SKP2-p21/p27 axis acts as a checkpoint pathway for the G1/S transition, only permitting $\mathrm{S}$ phase entry for cells whose mitogenic signalling outcompetes their load of DNA damage.

\section{Results}

NUCKS1 transcriptionally controls the SKP2-p21/p27 axis. To investigate whether NUCKS1 regulates the transcription of genes involved in cell cycle progression, we cross-compared a list of genes whose expression correlates with NUCKS1 mRNA in tumour samples and cell lines ${ }^{47}$, with genes whose promoter NUCKS1 binds in genome-wide ChIP-Seq ${ }^{16}$. This generated a list of 232 putative NUCKS1 target genes. Among them, we found several genes regulating the G1/S transition (e.g., SKP2, CCND1, CDK6, E2F3), DNA replication (e.g., PCNA), and the p53 pathway (e.g., MDM2). Gene Ontology (GO) biological processes enrichment analysis for the top hits, showing the best correlation with NUCKS1, reveals significant enrichment for genes associated with cell cycle progression (Supplementary Fig. 1A).

In a panel of the putative cell cycle targets, SKP2 displays the strongest and most reproducible downregulation upon NUCKS1 depletion (Supplementary Fig. 1B), and, given its role in cell cycle progression, DNA replication, and the $\mathrm{DDR}^{44,46,48,49}$, we focused on SKP2. To gain a more comprehensive understanding of NUCKS1's correlation with SKP2, we interrogated samples from The Cancer Genome Atlas (TCGA) database. Across a range of cancer types, mRNAs encoding NUCKS1 and SKP2 display a significant positive correlation (Fig. 1a). There is no such correlation between NUCKS1 and the housekeeping genes used as negative controls, B2M and GAPDH (Supplementary Fig. 1C). In particular, the correlation between NUCKS1 and SKP2 is most striking in glioblastoma, kidney renal papillary cell carcinoma, skin cutaneous melanoma, and uveal melanoma (Supplementary Fig. 1D).

To confirm binding of NUCKS1 at the SKP2 promoter $^{16}$, and to map the binding site, we designed ChIP-qPCR assays employing a panel of 10 primer sets spanning sequential regions of the SKP2 promoter (Fig. 1b). In these assays, we found that NUCKS1 displays specificity for the chromatin directly upstream of the SKP2 transcription start site (TSS), consistent with its role as a transcription factor (Fig. 1c).

Next, we tested the effect of NUCKS1 loss by siRNA-mediated depletion or CRISPR/Cas9-mediated deletion on SKP2 mRNA levels (Fig. 1d, Supplementary Fig. 1E). We found that loss of NUCKS1 reduces SKP2 gene expression across a cell line panel comprising three non-cancer cell lines (hTERT-immortalised bronchial epithelial cells: NBE1-hTERT; normal primary embryonic fibroblasts: TIG-1; hTERT-immortalised retinal epithelial cells: RPE1-hTERT), and six cancer cell lines (five colorectal cancer cell lines: HCT116, RKO, HT29, DLD1, CACO2; and osteosarcoma cells: U2OS) (Fig. 1d, Supplementary Fig. 1E). Loss of SKP2 occurs independently of the p53 pathway, the $\mathrm{Rb}$ pathway, the mitogenactivated protein kinase (MAPK) pathway, and microsatellite instability (MSI) status (Fig. 1d). Furthermore, NUCKS1 depletion reduces SKP2 protein levels and increases levels of SKP2's degradation targets, p21 and p27, confirming loss of SKP2 activity (Fig. 1e), and this is independent of p53 (Supplementary Fig. 1F, G). Consistent with the reduction in SKP2 levels, loss of NUCKS1 increases the stability of both p21 and p27, measured using cycloheximide chase assays (Supplementary Fig. 1H).

SKP2 mRNA levels are low in G0 and early G1, and increase during the G1/S transition ${ }^{50}$. To test G0/G1 cell cycle enrichment in NUCKS1-depleted cells (demonstrated in Fig. 3) as an indirect mechanism for SKP2 downregulation, we measured SKP2 levels in cells synchronised to G0/G1 before NUCKS1 depletion (Supplementary Fig. 1I). Under these conditions, loss of NUCKS1 still reduces SKP2 mRNA levels (Supplementary Fig. 1J), comparable with NUCKS1 depletion from asynchronous cells. 


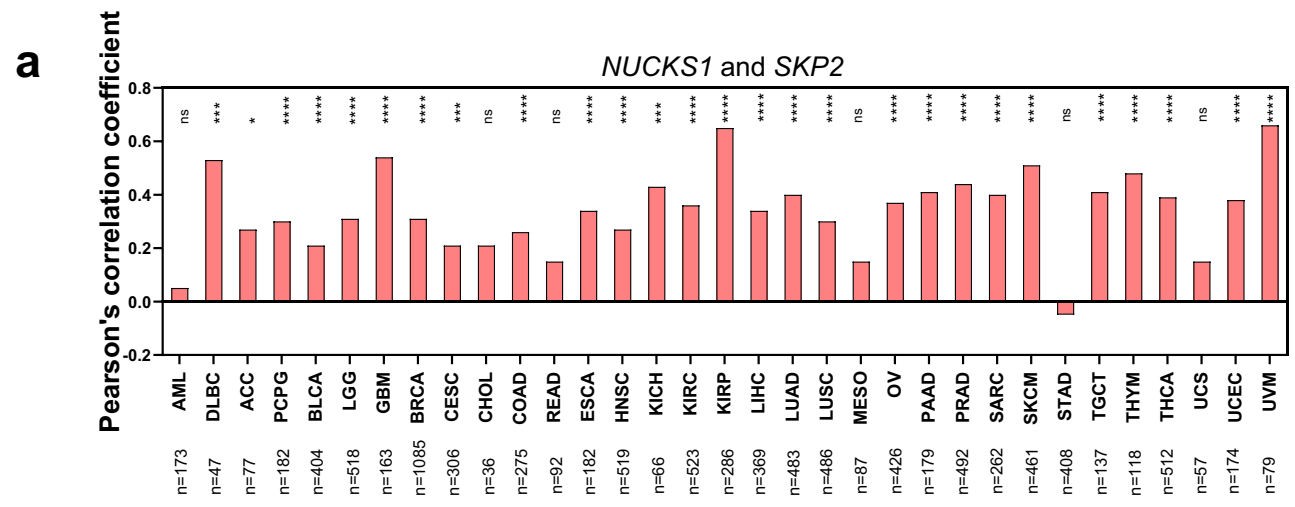

b

SKP2 locus - Chromosome 5: 36,151,989-36,196,849 (GRCh38)

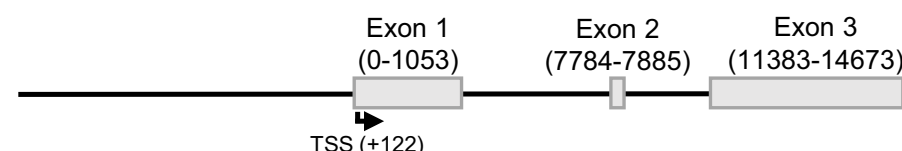

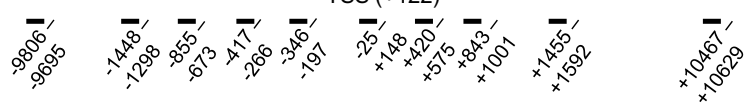

C
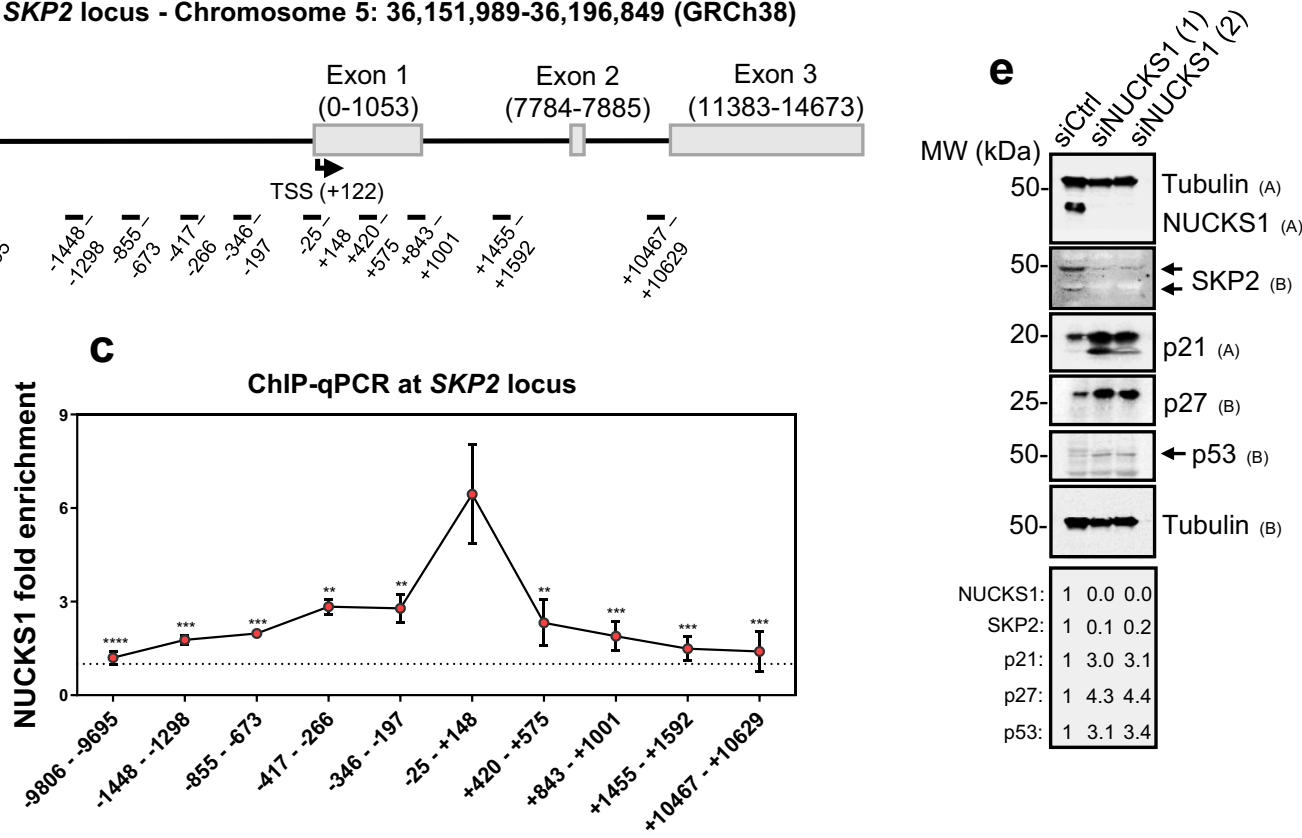

d

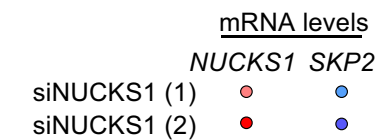

\begin{tabular}{lc} 
& mRNA levels \\
\cline { 2 - 2 } NUCKS1-KO (2) & 0 \\
NUCKS1-KO (3) & 0
\end{tabular}
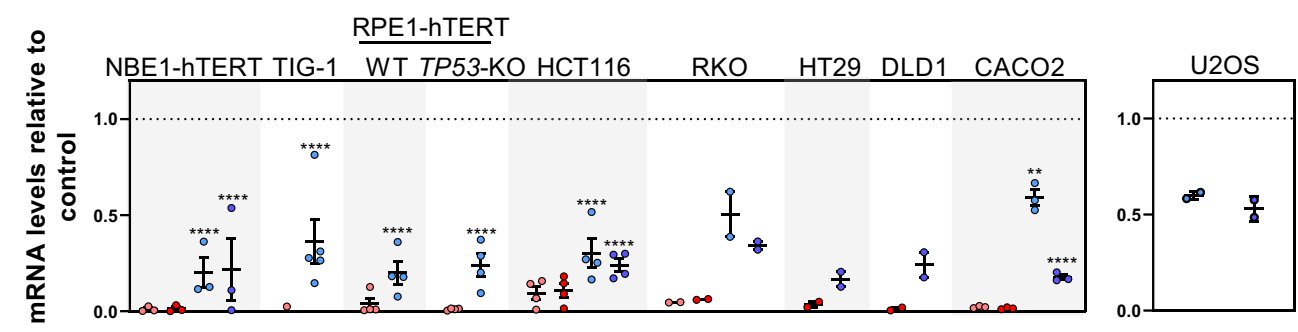

p53 pathway
Rb pathway
MAPK pathway
MSI

These results indicate that indirect cell cycle changes do not account for reduced levels of SKP2 in NUCKS1-depleted cells.

Altogether, these data identify $S K P 2$ as a transcriptional target of NUCKS1 and show that NUCKS1 regulates SKP2 expression independently of genetic background, and in multiple cellular contexts.
NUCKS1 levels and chromatin-binding are induced in late G1 to promote SKP2 expression and G1/S progression. To determine whether NUCKS1 itself is subject to cell cycle-dependent regulation, and to determine the point in the cell cycle during which NUCKS1 regulates SKP2, we measured protein levels of NUCKS1 and SKP2 over the course of the cell cycle after release 
Fig. 1 NUCKS1 transcriptionally controls the SKP2-p21/p27 axis. a Pearson's correlation (two-tailed) of NUCKS1 and SKP2 mRNAs from TCGA datasets, made using data from GEPIA2 ${ }^{79}$. b Map of human SKP2 promoter annotated with sequence positions of ChIP-qPCR primers. c ChIP-qPCR of NUCKS1 fold enrichment over lgG (dotted line) on the SKP2 promoter in U2OS cells. Ordinary one-way ANOVA with Dunnett's multiple comparisons test, using -25 +148 as a reference. Data are presented as mean \pm SEM from 3 independent experiments. $p$-values are in order as follows: 0.0001, 0.0004, 0.0007, $0.0058,0.0051,0.0016,0.0006,0.0002,0.0002$. d Left: RT-qPCR after control or siRNA-mediated NUCKS1 depletion. The dotted line denotes mRNA levels in siCtrl-treated cells. Right: RT-qPCR in two different clones of NUCKS1-KO U2OS cells. The dotted line denotes mRNA levels in WT U2OS cells. Left: One-way ANOVA with Sidak multiple comparisons test. Right: One-way ANOVA with Dunnett's multiple comparisons test. Data are presented as mean \pm SEM from 1-4 independent experiments. $p$-values are in order as follows: $<0.0001,<0.0001,<0.0001,<0.0001,<0.0001,<0.0001,<0.0001$, $0.0015,<0.0001$. e Western blot in control- or NUCKS1-depleted RPE1-hTERT cells. Representative of 3 independent experiments. MW: molecular weight, kDa: kilodaltons. Source data are provided as a source data file.

from G0/G1 synchronisation by contact inhibition. Using cyclin $\mathrm{A} 2$ as a marker for the onset of $\mathrm{S}$ phase ${ }^{51}$, we found that levels of NUCKS1 are low at the start of G1, increasing as cells progress into $S$ phase (Fig. 2a). The upregulation of SKP2 (but not NUCKS1) is driven partially ${ }^{52}$ by an increase in its mRNA levels, which is NUCKS1-dependent (Fig. 2b). Furthermore, we detected recruitment of NUCKS1 to chromatin following release from contact inhibition-mediated G0/G1 arrest, using PCNA and MLH1 - both of which are recruited to chromatin once $S$ phase has started ${ }^{53,54}$ as controls (Supplementary Fig. 2A). The major positive stimulus for $\mathrm{S}$ phase entry is provided by mitogens, which activate growth factor signalling 55 . We found that stimulation of cells with mitogens following $48 \mathrm{~h}$ of their withdrawal triggers the recruitment of NUCKS1 to chromatin, demonstrating a potential activation of NUCKS1 by mitogenic signalling (Fig. 2c).

Stimulation of NUCKS1 during G1 progression and by mitogens suggests an active role for NUCKS1 in S phase entry. To test this, we released control, NUCKS1-, or SKP2-depleted cells from G0/G1, and measured their ability to enter S phase. We found that siRNA-mediated NUCKS1 depletion substantially delays $S$ phase entry following G0/G1 release, phenocopying SKP2 loss (Fig. 2d, e; Supplementary Fig. 2B). Similarly, deletion of NUCKS1 from U2OS cells impairs S phase entry (Supplementary Fig. 2C).

Together, these results demonstrate that NUCKS1's recruitment to chromatin is stimulated by mitogens and increases during G1 progression. At the chromatin, NUCKS1 is required to induce SKP2 transcription and S phase entry.

NUCKS1 controls $S$ phase entry through the SKP2-p21/p27 axis. Next, we investigated the phenotypic impact of control of the SKP2-p21/p27 axis by NUCKS1. We found that CRISPR/ Cas9-mediated deletion of NUCKS1 enriches cells in G0/G1 phase of the cell cycle, with a concomitant reduction in replicating cells (Fig. 3a). This phenotype is reversed through overexpression of wildtype NUCKS1, but not by a DNA-binding defective mutant of NUCKS1 (in which the GRP motif is mutated to AAA), confirming that NUCKS1's DNA-binding activity is important for its role in cell cycle progression (Fig. 3b, Supplementary Fig. 3A). Furthermore, overexpression of NUCKS1 rescues cell cycle progression in NUCKS1-depleted HCT116 cells (Supplementary Fig. 3B, C), and NUCKS1 depletion delays cell cycle progression in TIG-1, NBE1-hTERT, and RPE1-hTERT cells (Supplementary Fig. 3D-F).

As a consequence, NUCKS1 deletion from U2OS cells (Fig. 3c), and NUCKS1 depletion from TIG-1 or NBE1-hTERT cells (Supplementary Fig. 3G, H), considerably reduce cellular proliferation. NUCKS1 depletion does not cause DNA damage, measured by alkaline comet assay (which detects single-strand breaks (SSBs) and DSBs) (Supplementary Fig. 3I) or $\gamma \mathrm{H} 2 \mathrm{AX} / 53 \mathrm{BP} 1$ immunofluorescence (Supplementary Fig. 3J), demonstrating that these phenotypes are not explained by DNA damage-induced quiescence.
We then tested whether the accumulation of p21/p27 in NUCKS1-depleted cells is due to the loss of SKP2. We found that overexpression of SKP2 in NUCKS1-depleted HCT116 (Fig. 3d) and A549 cells (Supplementary Fig. 3K) mostly induces degradation of the p21/p27 that accumulate in these cells. Consequently, overexpression of SKP2 in NUCKS1-depleted HCT116 (Fig. 3e, h) or A549 cells (Fig. 3f, i) largely rescues S phase entry. Similarly, co-depletion of SKP2's degradation targets, p21 or p27 (Fig. 3g, j; Supplementary Fig. 31, m, n), largely reverses cell cycle arrest.

Exploring these phenotypes further through proliferation assays, we found that SKP2 overexpression (Fig. 3k) or p21/p27 co-depletion (Fig. 3l) partially rescues the proliferation defects of NUCKS1-depleted cells. Finally, depletion of NUCKS1 from SKP2-depleted cells has no additional effect on proliferation, supporting the idea that SKP2 is a major determinant of NUCKS1's effect on proliferation (Supplementary Fig. 3O).

These results demonstrate that NUCKS1 controls p21/p27 levels, cell cycle progression, and proliferation through its transcriptional stimulation of the SKP2 gene, and identify the NUCKS1-SKP2-p21/p27 axis as a driving pathway for the G1/S transition.

Analysis of NUCKS1 binding at the SKP2 promoter. To more comprehensively understand the regulation of SKP2 by NUCKS1, we employed the electrophoretic mobility shift assay (EMSA), using a fluorescent SKP2 promoter probe (Fig. 4a). To do this, we started by purifying NUCKS1 from Sf9 insect cells, which preserves NUCKS1's post-translational modifications (Fig. 4b). We found that in-tact, phosphorylated NUCKS1 displays a low affinity for the SKP2 probe. However, dephosphorylation of NUCKS1 (using lambda phosphatase) increases the affinity of NUCKS1 for the SKP2 probe almost 10-fold (Fig. 4c, d).

Since a previous publication reported a GC-box as a potential NUCKS1 binding site ${ }^{16}$, since there is a GC-box within the sequence of peak NUCKS1 binding to the SKP2 promoter by ChIP-qPCR (Fig. 1c), and since recombinant NUCKS1 strongly binds the SKP2 EMSA probe, which contains a GC-box (Fig. 4a), we mutated this sequence and performed competition EMSAs to investigate whether NUCKS1 exhibits specificity for this site. We found that the interaction of NUCKS1 with the labelled SKP2 probe is readily outcompeted by a 100 -fold excess of unlabelled WT SKP2 probe, but not by an unlabelled mutant of the SKP2 probe with no GC-box (Fig. 4e, f).

Finally, we performed EMSAs using WT and NUCKS1-KO nuclear extracts, and found that nuclear extracts from WT cells display a much higher affinity for the SKP2 probe than extract from NUCKS1-KO cells (Fig. 4g, h).

Altogether, these results demonstrate that NUCKS1 directly interacts with the SKP2 promoter's DNA. The data suggest that this binding occurs via a GC-box in the SKP2 promoter, and may be regulated by the phosphorylation status of NUCKS1. 
a d

d $\quad$ siNUCKS1

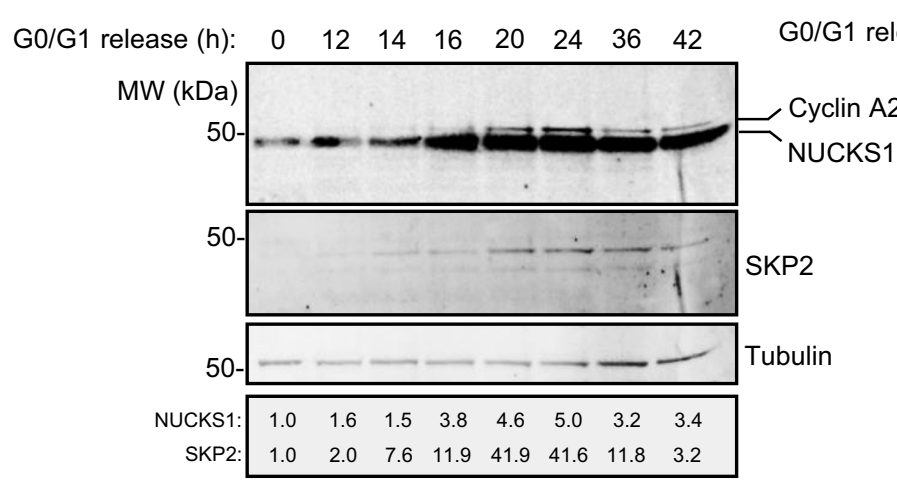

b
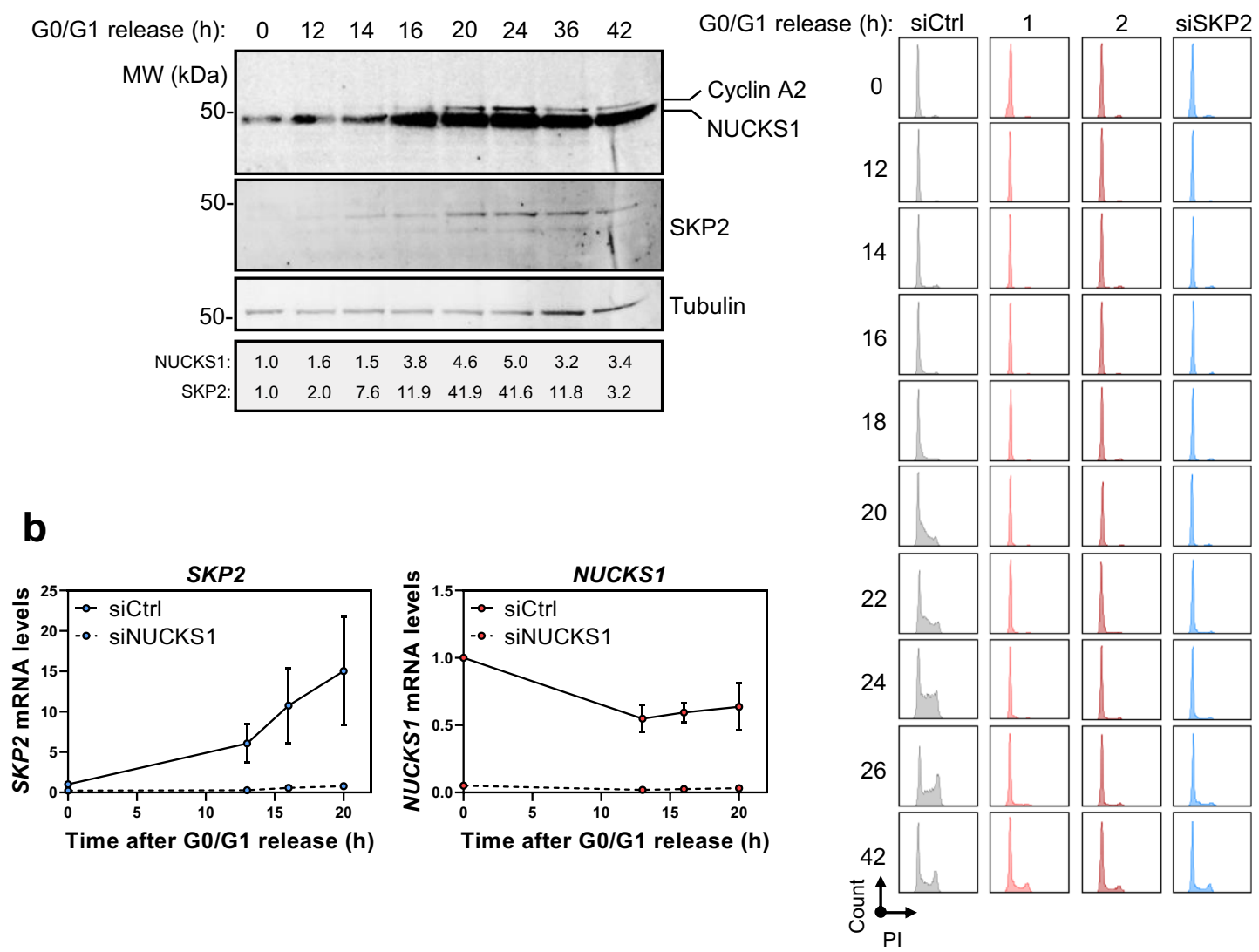

C

e

Mitogens (h): 010121620
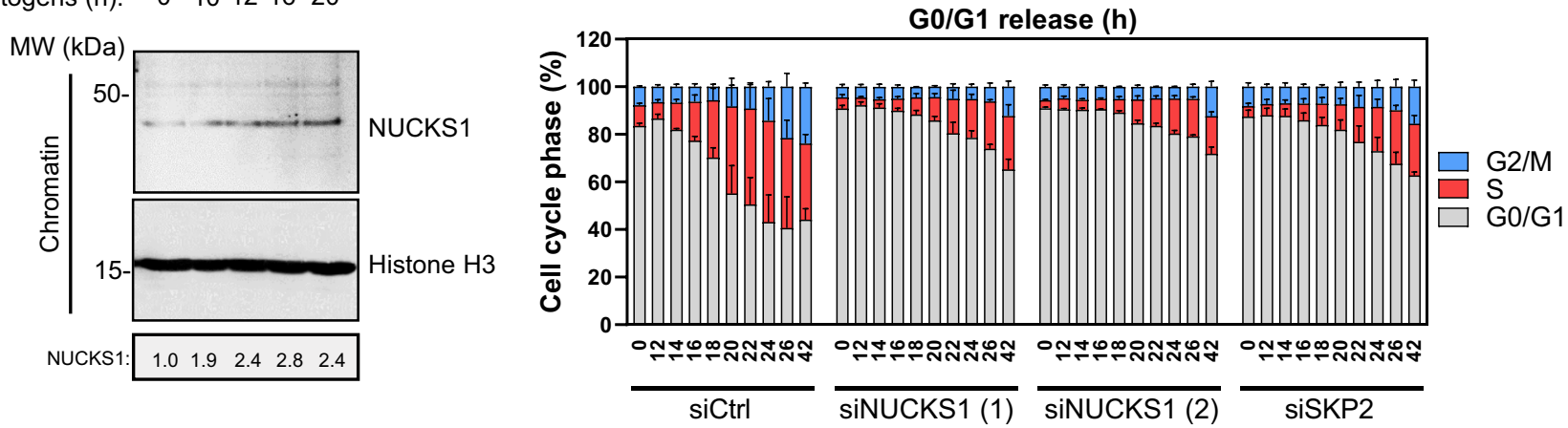

Fig. 2 NUCKS1 levels and chromatin-binding are induced in late G1 to promote SKP2 expression and G1/S progression. a Western blot in whole cell extracts of RPE1-hTERT cells synchronised to $\mathrm{GO} / \mathrm{G} 1$ by $72 \mathrm{~h}$ contact inhibition $(t=0)$ followed by re-plating at low density to release cells into $\mathrm{S}$ phase. Representative of 3 independent experiments. b RT-qPCR in NBE1-hTERT cells treated as in $\mathbf{a}$. Data are presented as mean \pm SEM from 3 independent experiments. c Western blot in the chromatin fraction of NBE1-hTERT cells starved of serum for $48 \mathrm{~h}(t=0)$ followed by mitogenic stimulation (15\% FBS) for the indicated periods of time. Representative of 2 independent experiments. d PI cell cycle profiles of control, NUCKS1-, or SKP2-depleted RPE1hTERT cells treated as in a. Representative of 3 independent experiments. e Quantification of d. Data are presented as mean \pm SEM from 3 independent experiments. MW: molecular weight, kDa: kilodaltons, PI: propidium iodide. Source data are provided as a source data file.

DNA damage inhibits the NUCKS1-SKP2 axis through p53dependent transcriptional repression. DNA damage activates an ATM/p53-dependent pathway to instigate cell cycle arrest, delay DNA replication, and accomplish DNA repair ${ }^{56}$. To determine whether this response involves NUCKS1 or SKP2, we analysed the NUCKS1-SKP2 axis following induction of DNA damage. In U2OS cells (which express WT TP53, encoding p53), treatment with the chemotherapeutic drug 5-fluorouracil (5-FU) markedly reduces NUCKS1 and SKP2 protein levels, with concomitant upregulation of p21 (controlled by both p53 and SKP2), and p27 (controlled by SKP2) (Fig. 5a). 5-FU treatment also abolishes occupancy of NUCKS1 at the SKP2 promoter (Fig. 5b), suggesting that downregulation of SKP2 is due to loss of NUCKS1 binding at its promoter. WT RPE1-hTERT cells treated with 5-FU similarly downregulate NUCKS1 and SKP2, and upregulate p21 and p27. However, this response is absent in TP53-KO RPE1hTERT cells, suggesting a role for $\mathrm{p} 53$ in DNA damage-mediated NUCKS1/SKP2 downregulation (Fig. 5c). Consistent with loss of 

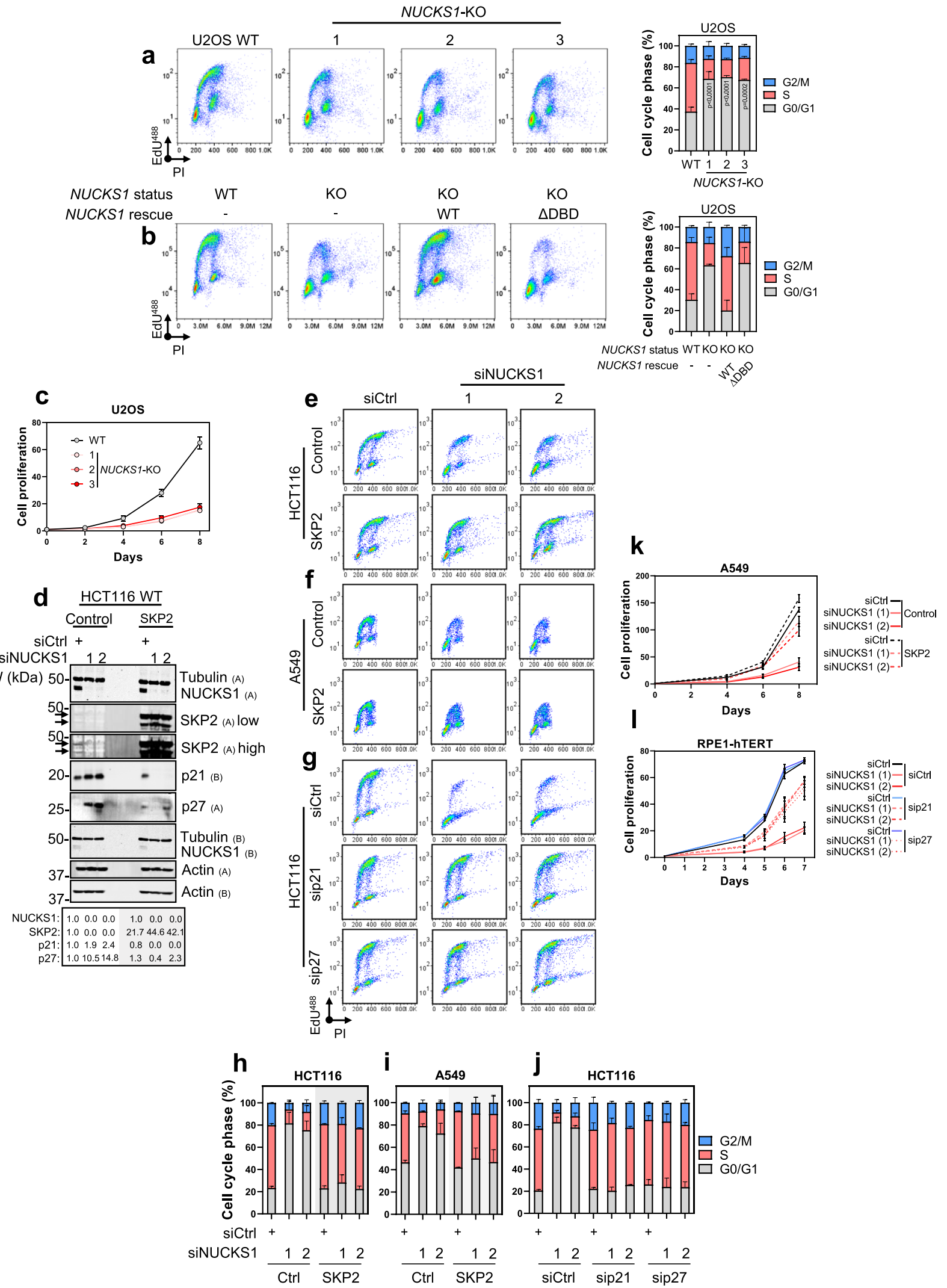

SKP2, the stability of p21 and p27 is extended in 5-FU-treated cells, revealed through chase assays with the translation inhibitor cycloheximide (Supplementary Fig. 4A).

To investigate this putative role for p53, we used RT-qPCR to measure the mRNA levels of NUCKS1 and SKP2 after treatment with 5-FU, IR, hydrogen peroxide $\left(\mathrm{H}_{2} \mathrm{O}_{2}\right)$, and camptothecin
(CPT), DNA-damaging agents which induce distinct DNA lesions. Upregulation of CDKN1A mRNA, encoding p21, was used as a control for p53 activation. We found that NUCKS1 and SKP2 transcripts are substantially reduced in response to all tested DNA-damaging agents (Fig. 5d). Consistent with Western blotting data, this is largely dependent on p53 (Fig. 5e-h). 
Fig. 3 NUCKS1 controls S phase entry through the SKP2-p21/p27 axis. a EdU/PI cell cycle profiles of WT U2OS cells and three different clones of NUCKS1-KO cells (left) and corresponding quantifications (right). Ordinary one-way ANOVA with Dunnett multiple comparisons test on S phase population. $\mathbf{b}$ EdU/PI cell cycle profiles of WT and NUCKS1-KO U2OS cells expressing the indicated variants of NUCKS1 (left) and corresponding quantifications (right). c Proliferation assay in WT U2OS cells and three different clones of NUCKS1-KO cells. d SKP2 overexpression largely rescues p21/ p27 accumulation in NUCKS1-depleted HCT116 cells, measured by Western blot. e SKP2 overexpression largely rescues HCT116 EdU/PI cell cycle profiles following treatment with control or NUCKS1 siRNA. f SKP2 overexpression largely rescues EdU/PI cell cycle profiles in A549 cells treated with control or NUCKS1 siRNA. $\mathbf{g}$ EdU/PI cell cycle profiles of HCT116 cells treated with control, p21, p27, or NUCKS1 siRNAs. h Quantification of HCT116 SKP2 cell cycle profiles in e. i Quantification of A549 SKP2 cell cycle profiles in $\mathbf{f}$. j Quantification of HCT116 p21/p27 cell cycle profiles in $\mathbf{g}$. k SKP2 overexpression partially rescues proliferation following NUCKS1 depletion in A549 cells. I Proliferation assay in RPE1-hTERT cells treated with control, NUCKS1, p21 or p27 siRNAs. In a (left), $\mathbf{b}$ (left), $\mathbf{d}, \mathbf{e}, \mathbf{f}$, and $\mathbf{g}$, data are representative of 3 (a, d) or 2 (b, e, $\mathbf{f}, \mathbf{g}$ ) independent experiments. In $\mathbf{a}$ (right), $\mathbf{b}$ (right), $\mathbf{c}, \mathbf{h}, \mathbf{i}, \mathbf{j}, \mathbf{k}$, and $\mathbf{I}$, data are presented as mean \pm SEM from $3(\mathbf{a}, \mathbf{c}, \mathbf{k}, \mathbf{I})$ or $2(\mathbf{b}, \mathbf{h}, \mathbf{i}, \mathbf{j})$ independent experiments. MW: molecular weight, kDa: kilodaltons, Pl: propidium iodide. Source data are provided as a source data file.

Induction of DNA damage also induces cell cycle changes which similarly depend on TP53 status (Supplementary Fig. 4B). These results demonstrate that DNA damage induces a p53 response, involving downregulation of NUCKS1 and SKP2, upregulation of p21 and p27, and cell cycle arrest.

Next, we sought to understand the mechanism underpinning p53-dependent downregulation of NUCKS1 and SKP2. Much p53-mediated transcriptional repression relies on activation of the DREAM transcriptional repression complex by p53-induced $\mathrm{p} 21^{57}$. To investigate whether this pathway is involved in the downregulation of NUCKS1 or SKP2, we treated WT and CDKN1A-knockout cells with 5-FU. As expected, transcripts of the p21-DREAM target $C C N B 1^{58}$, used as a positive control, are only reliably downregulated upon DNA damage in WT cells. However, transcripts of NUCKS1 and SKP2 are downregulated both in WT and CDKN1A-knockout cells, suggesting that NUCKS1 and SKP2 are not targets of the p21-DREAM pathway (Supplementary Fig. 4C-E).

Finally, we found that RNAi-mediated p53 depletion in TIG-1 cells, which express high endogenous p53 levels ${ }^{59}$ (Supplementary Fig. 4F), as well as deletion of TP53 from RPE1-hTERT cells (Supplementary Fig. 4G), increases NUCKS1 levels, suggesting that p53 may regulate NUCKS1/SKP2 expression both under basal conditions, as well as following p53 activation.

We propose that the p53-NUCKS1-SKP2-p21/p27 axis constitutes a checkpoint pathway for the G1/S transition, which may respond to DNA damage to prevent the replication of damaged DNA.

Copy number gain and p53 loss contribute to NUCKS1 and SKP2 overexpression in cancer. Transcriptional overexpression of NUCKS1 and SKP2 has been reported in numerous cancer types ${ }^{18,33-36,38,46,60-62}$. Although some reports, focused on specific cancer types, attribute this to increased copy number $33,37,39,46,61,63$, no pan-cancer analyses have been performed, and the full mechanisms underlying the upregulation remain poorly defined. Seeking to explore this further, we analysed NUCKS1 and SKP2 expression in TCGA datasets. NUCKS1 and SKP2 are overexpressed in most TCGA datasets, including many shared cancer types (Fig. 6a, b). Consistent with oncogenic functions for NUCKS1 ${ }^{39}$ and $S K P 2^{62}$, both genes are subjected to copy number increase in many cancers, while deletions are rare (Fig. 6c, d). These results confirm that increased copy number of NUCKS1 and SKP2 can contribute to their overexpression in cancer.

Because we found that p53 negatively regulates levels of NUCKS1 and SKP2 (Fig. 5), we investigated the effect of p53 mutation in cancer. To do so, we used p53-proficient vs. -deficient HCT116 cells, and expressed WT p53 as well as its DNA-binding mutants, R175H, R248W, and $\mathrm{R} 273 \mathrm{H}$, which frequently drive cancer ${ }^{64}$. We found that p53-deficient HCT116 cells have increased levels of both NUCKS1 and SKP2. Notably, overexpression of WT p53-but not its DNA-binding mutantssubstantially reduces NUCKS1/SKP2 levels (Fig. 6e). These results further support our finding that p53 negatively regulates levels of NUCKS1 and SKP2, and demonstrate that p53 mutants defective for DNA-binding lose the ability to repress NUCKS1/SKP2.

Finally, we asked whether p53 mutations also affect NUCKS1/SKP2 expression in cancer patients, using TCGA datasets. Consistent with our in vitro data, we found that mutation of $\mathrm{p} 53$ correlates with overexpression of NUCKS1/SKP2 in several cancer types (Fig. 6f).

Together, these results show that increased copy number, as well as p53 mutation, contribute to the overexpression of NUCKS1 and SKP2 in many cancers. This may enable cancer cells to proliferate in the absence of mitogenic stimulation, or in the presence of DNA damage.

\section{Discussion}

Here, we identify the NUCKS1-SKP2-p21/p27 axis as a cell cycle checkpoint pathway, which responds antagonistically to mitogen and DNA damage input to control S phase entry. In early G1 cells and in the absence of mitogens, NUCKS1 protein levels and chromatin retention are low, ensuring its inhibition in nonreplicating cells. NUCKS1 is upregulated and recruited to chromatin during G1/S progression, permitting NUCKS1 to stimulate the expression of SKP2, the F-box component of the SCF ${ }^{\mathrm{SKP} 2}$ ubiquitin ligase, leading to the degradation of p21/p27 and S phase entry. In contrast, DNA damage induces p53-dependent transcriptional repression of NUCKS1, leading to loss of SKP2 and upregulation of p21/p27 for cell cycle arrest. Some cancer cells hijack this mechanism, increasing NUCKS1/SKP2 copy numbers and transcriptionally upregulating NUCKS1 and SKP2 through p53 mutation. We propose that this may enable cancer cells to sustain proliferation, even in the absence of mitogens or in the presence of DNA damage (Fig. 7).

Our study identifies the SKP2-p21/p27 pathway as the second pathway transcriptionally controlled by NUCKS1, after the insulin receptor pathway ${ }^{16}$. However, the question of precisely how NUCKS1 regulates transcription remains unanswered. NUCKS1 is known to bind chromatin with higher affinity than naked DNA, does not bind ssDNA, and binds D-loops better than dsDNA ${ }^{19}$. NUCKS1 does not have a transcription activation domain, but promotes chromatin accessibility at-and recruits RNAPII to-its target promoters ${ }^{16}$. It is possible that NUCKS1 cooperates with other transcription factors to direct transcription; for example, NUCKS1 has been reported as an activator of NF- $\mathrm{KB}^{65}$. Since NF$\kappa B$ regulates SKP2 levels ${ }^{66}$, NUCKS1 may cooperate with NF- $\kappa B$ to control SKP2 expression. Nevertheless, future work will focus on characterising NUCKS1's interactome, to more deeply investigate its mechanism for transcriptional regulation.

Our EMSA data (Fig. 4) and others' ChIP-Seq data ${ }^{16}$ reveal that NUCKS1 displays affinity for the GC-box target sequence. 


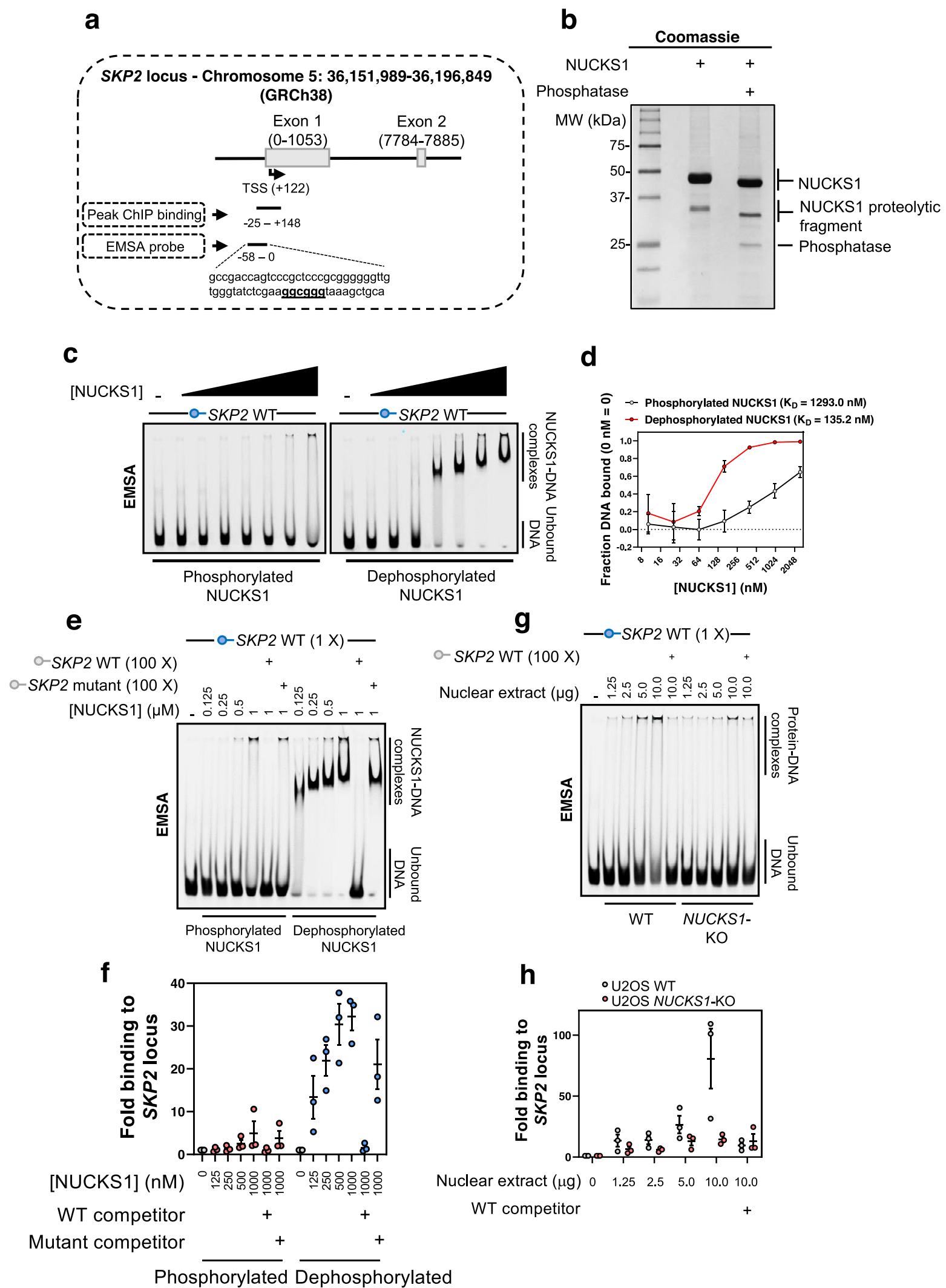

The sequence we identify, GGCGGG, is present twice within the 600 nucleotide SKP2 promoter, but is absent from the remaining $\sim 45,000$ nucleotides of the SKP2 gene, which may explain the specificity of NUCKS1 for the SKP2 promoter in vivo, and for other NUCKS1 targets more broadly. Going forward, research should focus on the structural basis of NUCKS1's interaction with the GC-box, and investigate whether NUCKS1 has multiple target DNA-binding sequences.

We show that p53 mediates the transcriptional downregulation of NUCKS1 in response to DNA damage (Fig. 5), but we do not fully characterise the mechanism. Binding of p53 at the NUCKS1 promoter, with enrichment following DNA damage, has been 
Fig. 4 Analysis of NUCKS1 binding at the SKP2 promoter. a Schematic showing sequence positions of the EMSA probe in relation to SKP2's transcription start site (TSS) and the region giving peak binding in our ChIP-qPCR assays. b Coomassie gel demonstrating NUCKS1 purification. Treatment with lambda phosphatase removes NUCKS1 phosphorylation and reduces its molecular weight. c Titration of phosphorylated or dephosphorylated NUCKS1 (10.24, 25.6, $64,160,400,1000,2500 \mathrm{nM}$ ) with the SKP2 promoter probe. d Quantification of $\mathbf{c}$. e Titration of phosphorylated or dephosphorylated NUCKS1 with the SKP2 promoter probe. In lanes $6 / 12$ and $7 / 13$, respectively, $100 \mathrm{X}$ molar quantity of unlabelled WT or mutant SKP2 probe were added as competition in binding reactions. $\mathbf{f}$ Quantification of $\mathbf{e} . \mathbf{g}$ Titration of WT or NUCKS1-KO U2OS nuclear extract with the SKP2 promoter probe. In lanes 6 and 11, 100 X molar quantity of unlabelled WT probe was added as competition in binding reactions. $\mathbf{h}$ Quantification of $\mathbf{g}$. In $\mathbf{b}$, $\mathbf{c}$, e, and $\mathbf{g}$, data are representative of 2 (b), 4 (c), or $3(\mathbf{e}, \mathbf{g})$ independent experiments. In $\mathbf{d}, \mathbf{f}$, and $\mathbf{h}$, data are presented as mean \pm SEM from 4 (d) or 3 (f, $\mathbf{h})$ independent experiments. MW: molecular weight, kDa: kilodaltons. Source data are provided as a source data file.

detected as part of genome-wide ChIP-Seq studies $^{32}$, and our data showing that the downregulation of NUCKS1 following DNA damage is independent of p21-DREAM (Supplementary Fig. 4) suggest that NUCKS1's repression may be a direct result of $\mathrm{p} 53$ binding. To explore this further, it would be useful to measure the rate of synthesis of new NUCKS1 transcripts, as well as the stability of NUCKS1 transcripts, to determine whether p53 controls NUCKS1's transcription itself or the stability of its mRNA. Complementary luciferase assays using the NUCKS1 promoter could also reveal whether p53 controls the activity of the NUCKS1 promoter. Furthermore, would mutation of a putative binding site for p53 in the NUCKS1 promoter alter NUCKS1 expression, DNA damage resistance, cell cycle progression, and proliferation? These experiments will form part of future studies.

NUCKS1 is the most post-translationally modified protein in the human proteome (for its size) and its major modification is phosphorylation ${ }^{23}$. NUCKS1 is phosphorylated by the G2/M cell cycle kinase CDK1 at S181, reducing NUCKS1 binding to $\mathrm{DNA}^{26,27}$, although the in vivo function of this phosphorylation is not completely understood. Interestingly, S181 phosphorylation of NUCKS1 could act to reset the level of chromatin-bound NUCKS1 for the daughter G1 phase, during which CDK1 activity is low, and explain the G1/S chromatin recruitment of NUCKS1 that we observe (Supplementary Fig. 2). Furthermore, the DDR kinase ATM promotes the indirect phosphorylation of NUCKS1 at S181 following DNA damage ${ }^{19,29,67}$. Therefore, ATMdependent NUCKS1 phosphorylation could provide a secondary mechanism to $\mathrm{p} 53$-dependent transcriptional repression, to ensure NUCKS1's removal from cell cycle promoters after DNA damage, and warrants investigation in the future. Notably, phosphorylation of NUCKS1 at S181 may also explain our EMSA data, which reveal a significant increase in DNA-binding affinity following NUCKS1 dephosphorylation (Fig. 4).

By stimulating the activity of RAD54, NUCKS1 promotes HR, the S/G2-specific DSB repair pathway ${ }^{19,22}$, demonstrating that NUCKS1 acts to maintain the fidelity of DNA replication. Consistent with this, we show that NUCKS1 levels remain high throughout $S$ phase and into G2 (Fig. 2). These findings raise a model in which NUCKS1 stimulates entry into $S$ phase and promotes the fidelity of the ensuing DNA replication through HR, after its role in $S$ phase entry is achieved. Notably, this function would mirror that of other G1/S factors, which boost both S phase entry and DNA repair, including E2F1 ${ }^{68}$ and SKP2 itself ${ }^{49}$.

In summary, our study identifies NUCKS1 as an important factor for the G1/S transition, placing NUCKS1 within the SKP2p21/p27 axis. Future studies will investigate NUCKS1's mechanism of transcriptional regulation, mechanisms for its regulation by posttranslational modification, and delve deeper into its roles in oncogenesis.

\section{Methods}

Cell culture. Cell lines (Supplementary Table 1) were cultured in DMEM (Life Technologies) with 15\% (TIG-1, NBE1-hTERT ${ }^{69}$ ) or $10 \%$ (U2OS, U2OS NUCKS1KO, RPE1-hTERT, RPE1-hTERT TP53-KO, RPE1-hTERT CDKN1A-KO ${ }^{70}$, HT29, A549 SKP2 doxycycline-inducible ${ }^{71}$, DLD1, RKO, HCT116, HCT116 TP53-KO ${ }^{72}$,
CACO2) $\mathrm{FBS}$, at $37^{\circ} \mathrm{C}$ in a humidified atmosphere with $5 \% \mathrm{CO}_{2}$. All cells tested negative for mycoplasma. For ionising radiation, treatments were performed using a GSR-D1 $137 \mathrm{Cs} \gamma$-irradiator (RPS Services) at a dose rate of $1.8 \mathrm{~Gy} / \mathrm{min}$.

siRNA and plasmid transfections. siRNA transfections were performed using Lipofectamine RNAiMAX, according to the manufacturer's instructions. Cells were transfected with $30 \mathrm{nM}$ siRNA for 3 days, unless otherwise indicated. siRNA sequences used are as follows:

siCtrl: Eurogentec, SR-CL000-005; siNUCKS1 (1): GAGGCGAUCUGGAAAG AAU; siNUCKS1 (2): GGCAUCUAAAGCAGCUUCU; siNUCKS1 (3' UTR): GCAGGAGGGACUAGAGAAAUU; siSKP2: GCUUCACGUGGGGAUGGGA; sip21: GAUGGAACUUCGACUUUGU; sip27: AAGGUUGCAUACUGAGCCA AG; sip53: AAGACUCCAGUGGUAAUCUAC.

Plasmid transfections were performed using Lipofectamine 3000, according to the manufacturer's instructions. Assays were performed $48 \mathrm{~h}$ after plasmid transfection. Plasmids used in the study are listed in Supplementary Table 2.

CRISPR-Cas9 genome editing. NUCKS1 CRISPR/Cas9 KO plasmid (sc-413018) and NUCKS1 HDR plasmid (sc-413018-HDR) were co-transfected into early passage U2OS cells. Cells were treated with $5 \mu \mathrm{g} / \mathrm{ml}$ puromycin for 3 days to select successfully-transfected cells, and seeded as single cells. Colonies were expanded and successful clones were confirmed using RT-qPCR and Western blotting.

Western blotting. Whole cell extracts were prepared as described previously $y^{73}$ Nuclear/chromatin fractionations were performed as described previously ${ }^{74}$. Proteins were resolved using SDS-PAGE and transferred onto Immobilon-FL PVDF membranes (Millipore). Membranes were blocked using Odyssey blocking buffer (Li-Cor) and blotted using the antibodies indicated in Supplementary Table 3. Western blot detection was performed using the Odyssey image analysis system (Li-Cor Biosciences). Analysis and quantification were performed using Image Studio Lite Ver 5.2

RT-qPCR. Total RNA was extracted using the RNeasy kit (QIAGEN). Reverse transcription was performed using the SuperScript II Reverse Transcriptase kit (Thermofisher). RT-qPCR was performed using Fast SYBR Green Master Mix (Thermofisher) and the 7500 Fast Real-Time PCR System (Applied Biosystems), with the comparative CT method for quantification. Analysis was performed using 7500 Software v2.0.6. Reference genes used for RT-qPCR are B2M/GAPDH/TBP. Primer sequences are listed in Supplementary Table 4.

Protein expression and purification. Production of baculoviral particles, infection of Sf9 cells, and expression of recombinant protein was performed as described previously ${ }^{75}$. Mid log phase Spodoptera frugiperda (Sf9) cells $\left(2 \times 10^{6} / \mathrm{ml}\right)$ were transfected with pDEST53-NUCKS1 bacmid using Cellfectin II transfection reagent in a 6-well plate format, according to the manufacturer's protocol. Following incubation for 5 days at $27^{\circ} \mathrm{C}$, medium containing P0 baculovirus was collected and stored at $4{ }^{\circ} \mathrm{C}$, protected from light. Two sequential rounds of virus amplification were performed to generate higher titre $\mathrm{P} 2$ baculovirus stocks. Sf9 cells were infected with P2 virus $(120 \mu \mathrm{g} / 50 \mathrm{ml} \mathrm{Sf} 9)$, and incubated at $27^{\circ} \mathrm{C}$ for 3 days on an orbital shaker. Cells were harvested by centrifugation $\left(900 \mathrm{~g}, 20 \mathrm{~min}, 4^{\circ} \mathrm{C}\right)$, washed with PBS, pelleted again, and then stored at $-80^{\circ} \mathrm{C}$. Cell pellets were resuspended in buffer A ( $50 \mathrm{mM}$ HEPES $\mathrm{pH} 7.5,300 \mathrm{mM} \mathrm{NaCl}, 5 \%(\mathrm{w} / \mathrm{v})$ glycerol) supplemented with $1 \mathrm{mM}$ TCEP, and 1:500 (v/v) protease inhibitor cocktail (Sigma-Aldrich, P8849) (12 $\mathrm{ml}$ buffer A per 100-ml culture cell pellet), and lysed by sonication, followed by incubation with Benzonase ( $20 \mathrm{U} / \mu \mathrm{l})$ for $30 \mathrm{~min}$ on ice. Cell lysate were clarified by centrifugation, and supernatant passed through a $0.45-\mu \mathrm{m}$ syringe filter. The supernatant was then supplemented with $5 \mathrm{mM}$ imidazole prior to loading onto a 1-ml HisTrap column (GE Healthcare) attached to an AKTA system at $1 \mathrm{ml} / \mathrm{min}$ After sample loading, the column was washed with buffer A containing $5 \mathrm{mM}(10$ column volumes (CV)) and $50 \mathrm{mM}$ imidazole (10CV). NUCKS1 was eluted with a linear $50-250 \mathrm{mM}$ imidazole gradient $(20 \mathrm{CV})$ and $0.5 \mathrm{ml}$ fractions were collected. $\mathrm{His}_{6}$-tagged NUCKS1-containing fractions were pooled, and dialysed against storage buffer ( $50 \mathrm{mM}$ Tris $\mathrm{pH} 7.5,150 \mathrm{mM} \mathrm{NaCl}, 5 \%(\mathrm{w} / \mathrm{v})$ glycerol, $0.5 \mathrm{mM} \mathrm{DTT})$ To improve purity, His $_{6}$-tagged NUCKS1 was further purified by size-exclusion chromatography. $1.5 \mathrm{mg}$ of HisTrap-purified NUCKS1 was diluted to $200 \mu \mathrm{l}$ in 


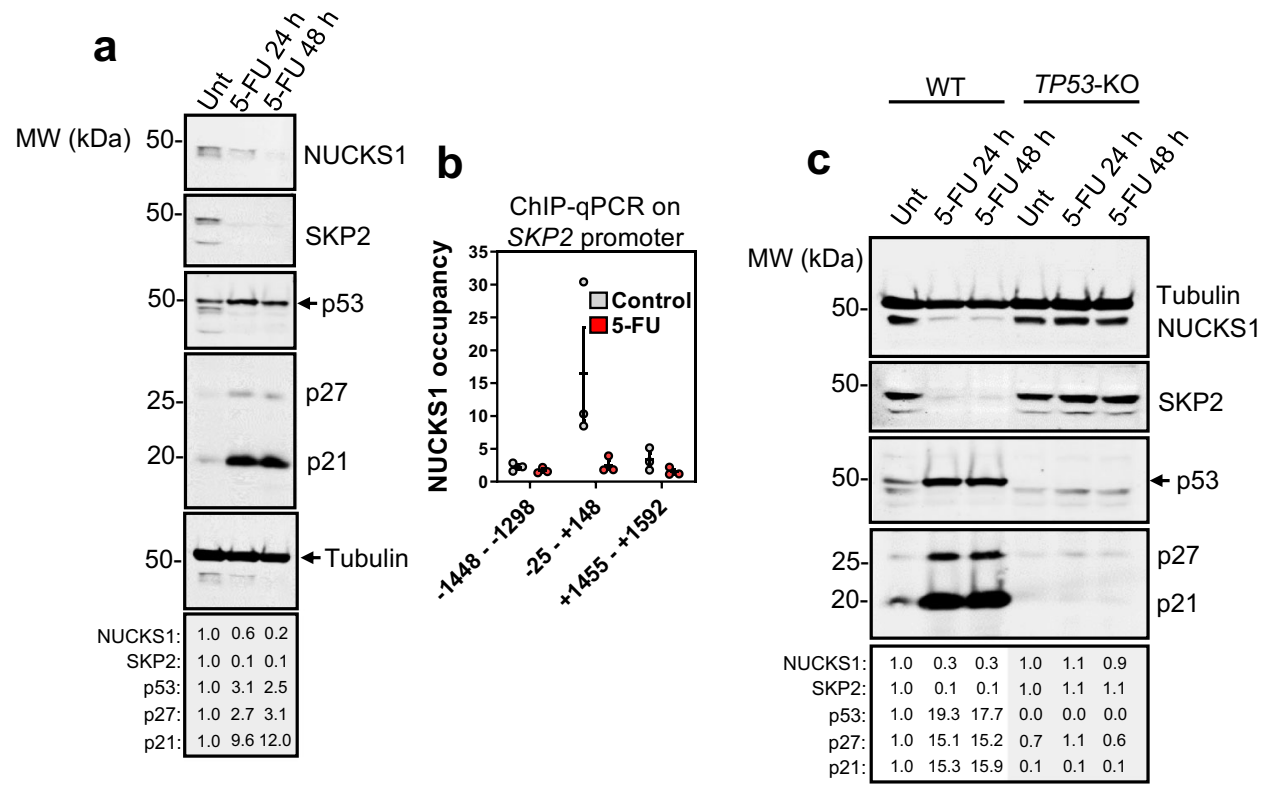

d
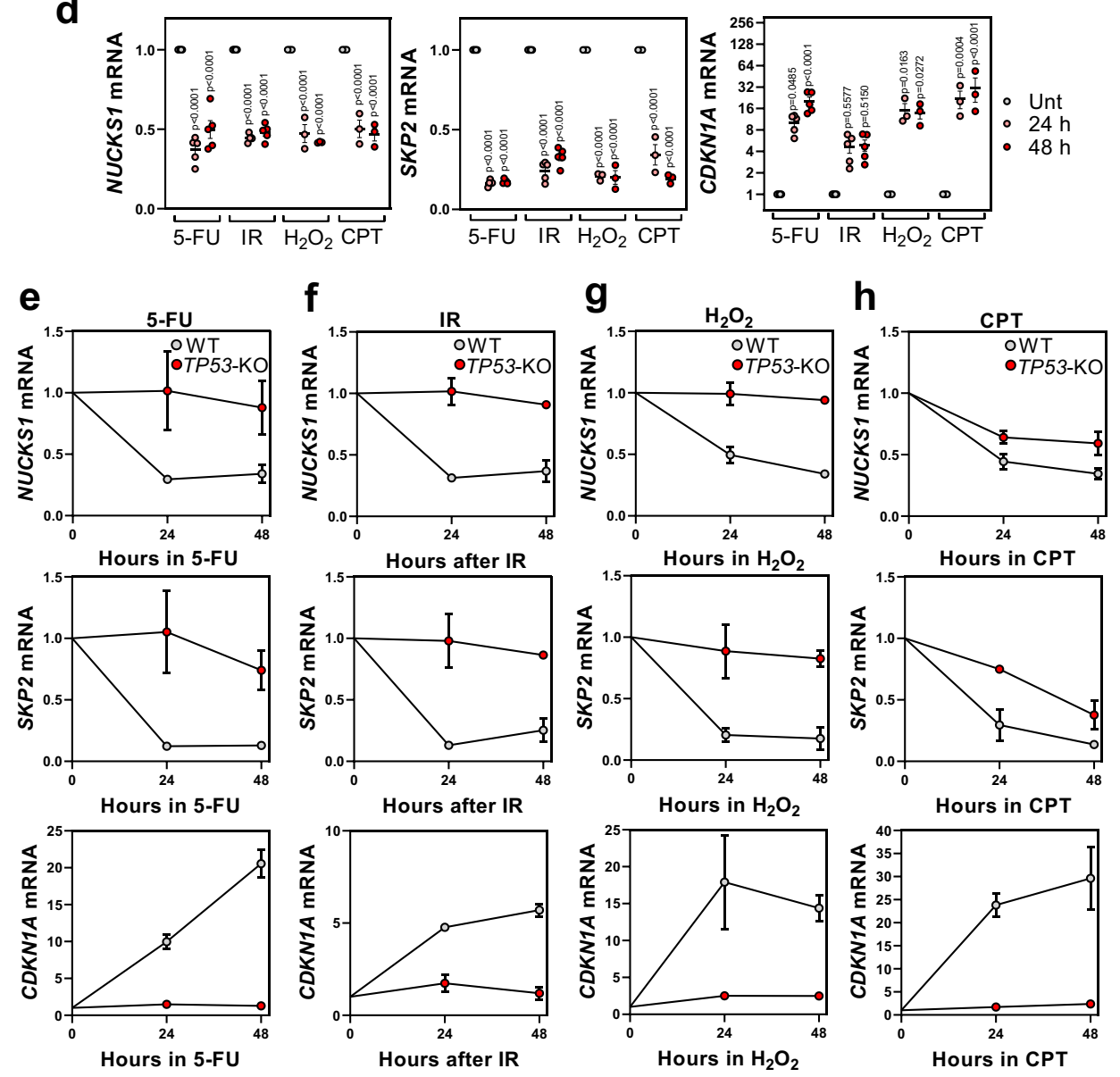

Fig. 5 DNA damage inhibits the NUCKS1-SKP2 axis through p53-mediated transcriptional repression. a Western blot in WT U2OS cells treated with $50 \mu \mathrm{M} 5$-FU for 24 or $48 \mathrm{~h}$. b ChIP-qPCR of NUCKS1 fold enrichment over lgG (=1) on the SKP2 promoter in U2OS cells after treatment with control or $50 \mu \mathrm{M}$ 5-FU for $48 \mathrm{~h}$. c Western blot in RPE1-hTERT WT and TP53-KO cells treated with $10 \mu \mathrm{M} \mathrm{5-FU}$ for 24 or $48 \mathrm{~h}$. d RT-qPCR in WT RPE1-hTERT cells treated with 5-FU $(10 \mu \mathrm{M}), \mathrm{IR}(4 \mathrm{~Gy}), \mathrm{H}_{2} \mathrm{O}_{2}(200 \mu \mathrm{M})$, or CPT $(100 \mathrm{nM})$ for 24 or $48 \mathrm{~h}$. Ordinary two-way ANOVA with Dunnett's multiple comparisons test. e RT-qPCR after 5-FU (10 $\mu$ M) in RPE1-hTERT WT or TP53-KO cells. f RT-qPCR after IR (4 Gy) in RPE1-hTERT WT or TP53-KO cells. $\mathbf{g}$ RT-qPCR after $\mathrm{H}_{2} \mathrm{O}_{2}(200 \mu \mathrm{M})$ in RPE1-hTERT WT or TP53-KO cells. h RT-qPCR after CPT (100 nM) in RPE1-hTERT WT or TP53-KO cells. In a and $\mathbf{c}$, data are representative of 3 independent experiments. In $\mathbf{b}, \mathbf{d}, \mathbf{e}, \mathbf{f}, \mathbf{g}$, and $\mathbf{h}$, data are presented as mean \pm SEM from 3 (b, h), 3-5 (d) or 2 (e-g) independent experiments. MW: molecular weight, kDa: kilodaltons. Source data are provided as a source data file. 
a

b
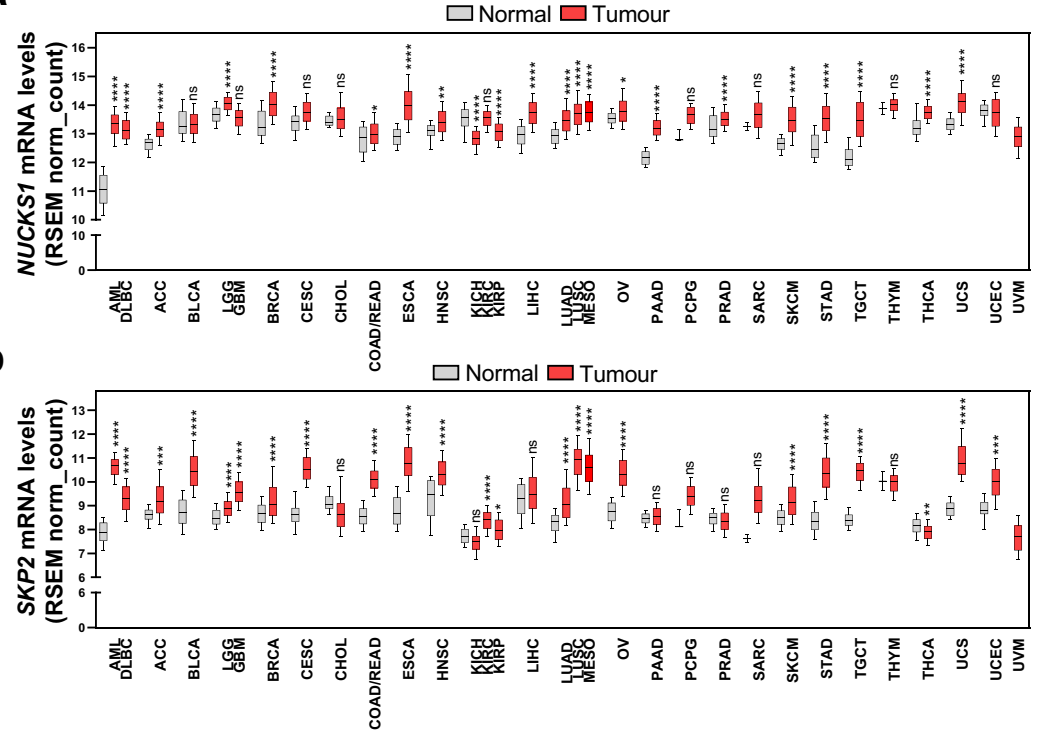

C

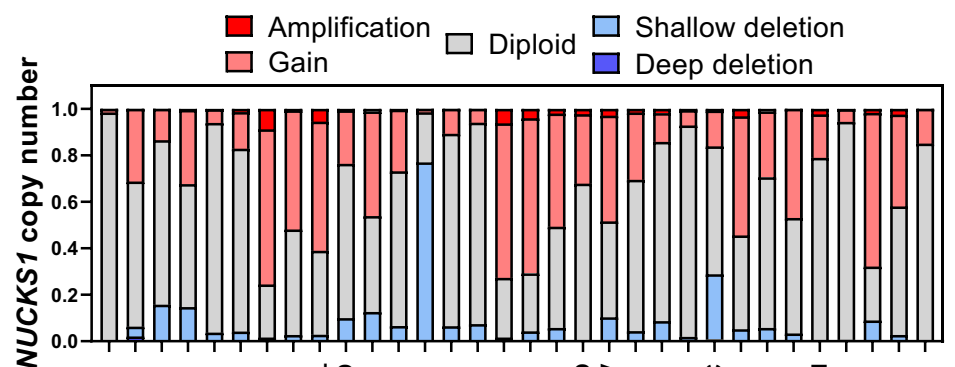

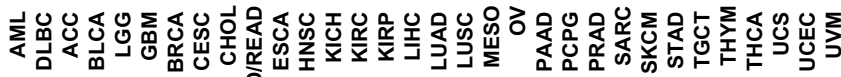

d

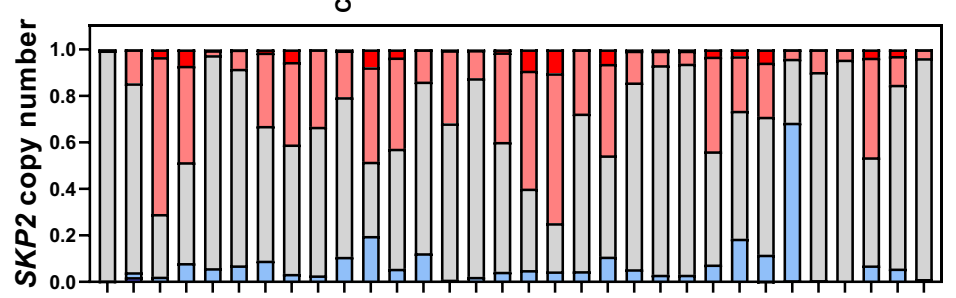

MW (kDa)

e
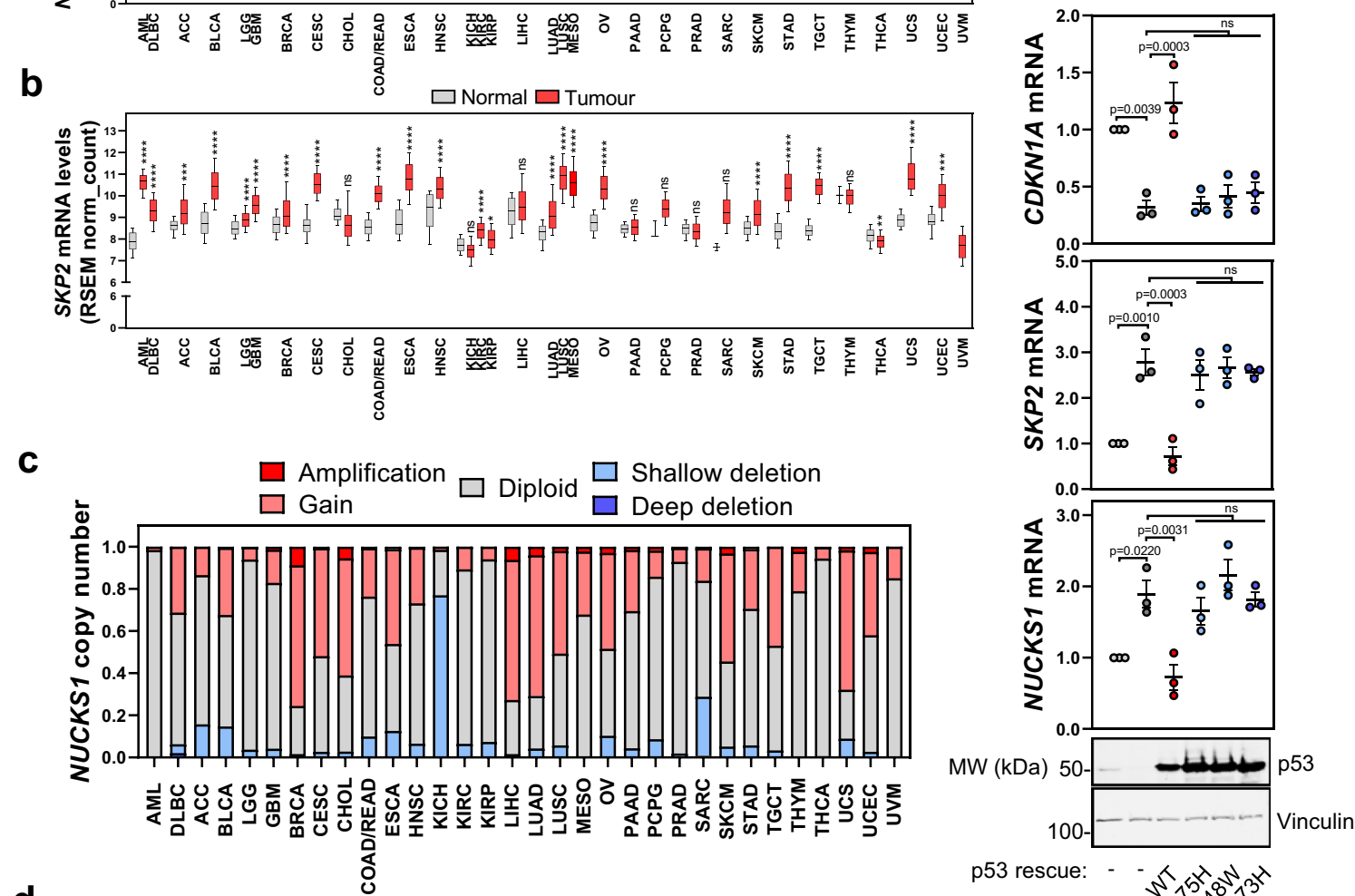

p53 rescue: - - $2 \sqrt{2}$

p53 status: WT TP53-KO

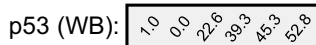

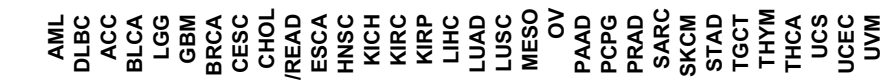

高

f ESCA

LUSC

UCEC

BRCA

METABRIC
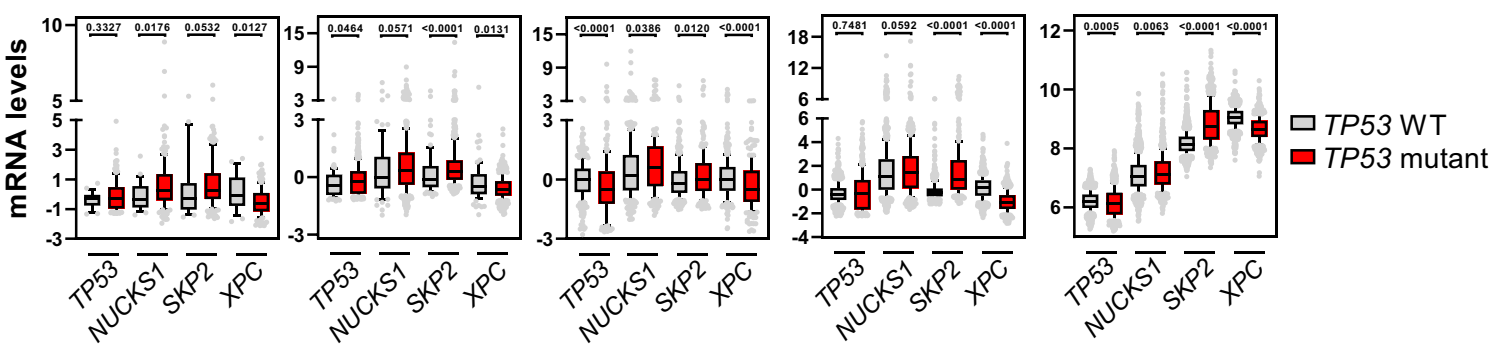

storage buffer and loaded onto a Superdex 200 HR 10/30 column (GE Healthcare, Little Chalfont, UK), and $0.5 \mathrm{ml}$ fractions collected.

Immunofluorescence. Cells seeded on coverslips were subjected to pre-extraction in a buffer containing $10 \mathrm{mM}$ HEPES, $100 \mathrm{mM} \mathrm{NaCl}, 0.3 \mathrm{M}$ sucrose, $3 \mathrm{mM} \mathrm{MgCl}_{2}$, and $0.1 \%$ Triton $\mathrm{X}-100$ for two minutes, washed twice using a buffer containing
$10 \mathrm{mM}$ HEPES, $100 \mathrm{mM} \mathrm{NaCl}, 0.3 \mathrm{M}$ sucrose, and $3 \mathrm{mM} \mathrm{MgCl}_{2}$, and then fixed in $4 \%$ formaldehyde for 15 min on ice. Cells were blocked overnight in $5 \%$ BSA at $4{ }^{\circ} \mathrm{C}$, and primary antibodies (indicated in Supplementary Table 3) were diluted in $2.5 \% \mathrm{BSA}$ and incubations performed for $1 \mathrm{~h}$ at RT. Cells were washed in PBS, incubated in secondary antibodies (indicated in Supplementary Table 3 ) for $1 \mathrm{~h}$ at RT, and stained with DAPI. Microscopy was performed using the Nikon NiE and quantification was performed using CellProfiler ${ }^{76}$. 
Fig. 6 Copy number gain and p53 loss contribute to NUCKS1 and SKP2 overexpression in cancer. a NUCKS1 expression in normal vs. tumour tissue, using data from UCSC Xena ${ }^{80}$. In $\mathbf{a}$ and $\mathbf{b}$, box plots show median values along with 25/75\% (box) and 10/90\% (whiskers). Statistics were analysed using Kruskal Wallis with Dunn's post test. $p$-values are in order as follows: $<0.0001,<0.0001,<0.0001,>0.9999,<0.0001,0.1198,<0.0001,>0.9999$, $>0.9999,0.0148,<0.0001,0.0019,<0.0001,>0.9999,<0.0001,<0.0001,<0.0001,<0.0001,<0.0001,0.0187,<0.0001,0.6879,<0.0001,>0.9999$, $<0.0001,<0.0001,<0.0001,>0.9999,<0.0001,<0.0001,>0.9999$. b SKP2 expression in normal vs. tumour tissue, using data from UCSC Xena 80 . $p$-values are in order as follows: <0.0001, <0.0001, 0.0002, <0.0001, <0.0001, <0.0001, <0.0001, <0.0001, >0.9999, <0.0001, <0.0001, <0.0001, $>0.9999,<0.0001,0.0476,0.3634,<0.0001,<0.0001,<0.0001,<0.0001,>0.9999,>0.9999,>0.9999,0.3562,<0.0001,<0.0001,<0.0001,>0.9999$, $0.0023,<0.0001,0.0006$. c NUCKS1 copy number changes in cancer, using data from TCGA PanCancer Atlas datasets in CBioPortal 81,82 . d SKP2 copy number changes in cancer, using data from TCGA PanCancer Atlas datasets in CBioPortal ${ }^{81,82}$. e Western blot and RT-qPCR in p53 proficient vs. deficient HCT116 cells expressing indicated variants of p53. Ordinary one-way ANOVA with Tukey's multiple comparisons test. Upper: data are presented as mean + / - SEM from 3 independent experiments. Lower: data are representative of 3 independent experiments. ns p-values are in order as follows NUCKS1: 0.8983, 0.8478, 0.9995; SKP2: 0.9412, 0.9984, 0.9786; CDKN1A: 0.9999, 0.9802, 0.9334. f Analysis of TP53, NUCKS1, SKP2 or XPC (used as a positive control for p53 activity) mRNA levels in WT vs. TP53 mutant tumours, using PanCancer Atlas or METABRIC datasets in CBioPortal81,82. Units: log RNA Seq V2 RSEM (ESCA-BRCA) and mRNA expression microarray (METABRIC). Box plots show median values along with 25/75\% (box) and 10/90\% (whiskers) and outliers. Two-tailed Mann-Whitney test. ESCA p53 WT $n=24$ patients, p53 mutant $n=157$ patients. LUSC p53 WT $n=79$ patients, p53 mutant $n=402$ patients. UCEC p53 WT $n=323$ patients, p53 mutant $n=192$ patients. BRCA p53 WT $n=717$ patients, p53 mutant $n=347$ patients. METABRIC p53 WT 1245 patents, p53 mutant $n=659$ patients. MW: molecular weight, kDa: kilodaltons. Source data are provided as a source data file.

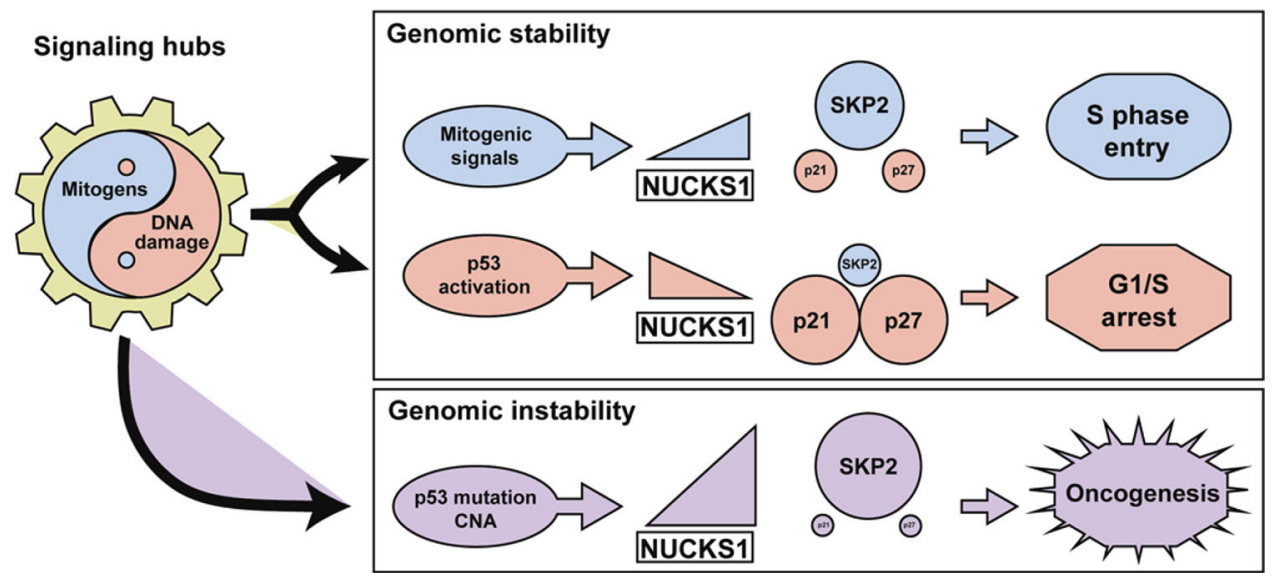

Fig. 7 Model depicting the role for NUCKS1 in the S phase entry decision. The NUCKS1-SKP2-p21/p27 axis constitutes a signalling hub which integrates the opposing cell cycle signals, mitogens and DNA damage. Mitogens stimulate binding of NUCKS1 to the SKP2 promoter, SKP2 expression, p21/p27 degradation, and S phase entry. DNA damage induces p53-dependent repression of NUCKS1, leading to SKP2's transcriptional downregulation, upregulation of p21/p27, and cell cycle arrest. Some cancer cells increase NUCKS1/SKP2 copy number and mutate p53, leading to NUCKS1 and SKP2 overexpression.

Site-directed mutagenesis. NUCKS1's GRP DNA-binding motif was mutated to AAA using the Phusion Site-Directed Mutagenesis Kit (Thermofisher), with primers as follows: gcctttgaagctgtggcggcagccactttccctttgcc/ggcaaagggaaagtggctgccgccacagcttcaaaggc. Mutant NUCKS1 was validated by sequencing.

Comet assays. Alkaline comet assays were performed as described previously ${ }^{77}$, using the Nikon NiE and Andor Komet7.1 software.

Proliferation assays. Cells were seeded at day 0 , treated as indicated, and viable cells were counted at indicated days, using Trypan Blue staining (Life Technologies) and the Countess ${ }^{\mathrm{m}}$ Automated Cell Counter (Thermo Fisher Scientific).

EMSAs. For NUCKS1 EMSAs, recombinant NUCKS1 was dephosphorylated using lambda phosphatase (100 units/1 $\mu \mathrm{g}$ of recombinant NUCKS1), in the presence of Protein MetalloPhosphatases buffer and $\mathrm{MnCl}_{2}(1 \mathrm{mM})$, for $90 \mathrm{~min}$ at $30^{\circ} \mathrm{C}$, followed by addition of phosphatase inhibitor cocktail (50x, Merck Millipore). Consequently, binding reactions using indicated quantities of intact or dephosphorylated NUCKS1, or WT or NUCKS1-KO nuclear extract, were set up in the presence of binding buffer ( $20 \mathrm{mM}$ Tris- $\mathrm{HCl} \mathrm{pH} \mathrm{7.5,100} \mathrm{mM} \mathrm{KCl,} 0.2 \% \mathrm{NP}-40$, $20 \%$ glycerol, $2 \mathrm{mM}$ DTT) supplemented with $50 \mathrm{ng}$ or $1 \mu \mathrm{g}$ salmon sperm DNA (for pure protein and nuclear extract, respectively). $25 \mathrm{nM}$ double-stranded probes were added, and reactions were incubated for $15 \mathrm{~min}$ at $37^{\circ} \mathrm{C}$ before loading on native 6\% PAGE gels at $150 \mathrm{~V}$ for $50 \mathrm{~min}$. Gels were imaged using the Odyssey image analysis system (LiCor Biosciences). The double-stranded sequence of the SKP2 probes used in EMSAs were Gccgaccagtcccgctcccgcggggggttgtgggtatctcgaaggcgggtaaagctgca (WT SKP2 probe) and GccgaccagtcccgctcccgcggggggttgtgggtatctcgaaAAAAAAtaaagctgca (mutant SKP2 probe). The WT probe was IRDye- 800 fluorescence-labelled. In competition assays, unlabelled probes were included in binding reactions at 100-times the concentration of labelled probes. Analysis and quantification were performed using Image Studio Lite Ver 5.2.

ChIP-qPCR. ChIP was performed as previously described ${ }^{59}$, using U2OS cells fixed in $1 \%$ formaldehyde for $15 \mathrm{~min}$, ensuring sonication fragments between 100 and $500 \mathrm{bp}$, and using $5 \mu \mathrm{g}$ anti-NUCKS1 antibody (ProteinTech 12023-2-AP) or $5 \mu \mathrm{g}$ normal rabbit IgG (SantaCruz sc2027). Primers used for ChIP-qPCR are listed in Supplementary Table 4.

Flow cytometry. For propidium iodide staining, trypsinised cells were fixed in cold $70 \%$ ethanol for $30 \mathrm{~min}$ on ice. Cells were then centrifuged at $250 \mathrm{~g}$ for $5 \mathrm{~min}$ and resuspended in PBS with $0.5 \mu \mathrm{g} / \mathrm{ml}$ RNAseA and $10 \mu \mathrm{g} / \mathrm{ml}$ propidium iodide, before incubation for $15 \mathrm{~min}$ at $37^{\circ} \mathrm{C}$. For EdU/PI staining, the Click-iT ${ }^{\mathrm{mt}}$ Plus EdU Alexa Fluor ${ }^{\mathrm{mw}} 488$ Flow Cytometry Assay Kit was used, according to the manufacturer's instructions.

The BD FACSCalibur ${ }^{\mathrm{Tm}}$ (BD Biosciences) or CytoFLEX (Beckman Coulter) machines were used for sample acquisition. FlowJo v10.6.1 and ModFit LT 4.1.7 were used for analysis.

Bioinformatics. Bioinformatics screens for putative transcriptional targets of NUCKS1, as outlined in Supplementary Fig. 1A, were performed using the SEEK database $^{47}$ (http://seek.princeton.edu/). The SEEK database was used to generate lists of the 1000 genes correlating most positively with NUCKS1 across 15 different cancer types, with three sample types per cancer (cancer tissue, tumour tissue, or cell line). This generated 45 lists of 1,000 genes, which were subsequently, independently, filtered through NUCKS1-interacting promoters in ChIP-Seq data ${ }^{16}$ and ranked (Supplementary Fig. 1A). The GO biological processes enrichment presented in Supplementary Fig. 1A was generated using Genecodis $3^{78}$ (http://genecodis.cnb.csic.es). Figure 1a and supplementary Fig. 1C and 1D were 
generated using data from GEPIA2 ${ }^{79}$ (http://gepia2.cancer-pku.cn/\#index). Figure $6 a$ and $b$ were generated using data from UCSC XENA (https://xena.ucsc.edu/), using RSEM norm count values from GTEX/TCGA normal datasets, and TCGA tumour datasets ${ }^{80}$. Figure $6 c, d$ and $\mathrm{f}$ were generated using data from CBioPortal ${ }^{81,82}$ (https://www.cbioportal.org/).

Statistical analyses. Statistical tests, indicated in figure legends, were performed using GraphPad Prism 8.

Reporting summary. Further information on research design is available in the Nature Research Reporting Summary linked to this article.

\section{Data availability}

The data that support this study are available from the corresponding authors upon reasonable request. Supplementary Fig. 1A uses NUCKS1 ChIP-Seq data ${ }^{16}$ (accession code GSE58100), the SEEK database ${ }^{47}$ (http://seek.princeton.edu/) and Genecodis $3^{78}$ (http://genecodis.cnb.csic.es). Figure 1a and Supplementary Fig. 1C and 1D were generated using data from GEPIA2 ${ }^{79}$ (http://gepia2.cancer-pku.cn/\#index). Figure 6a and b were generated using data from UCSC XENA (https://xena.ucsc.edu/), using RSEM norm count values from GTEX (https://gtexportal.org/home/) and TGCA (https:// portal.gdc.cancer.gov/) normal datasets, and TCGA tumour datasets ${ }^{80}$. Figure $6 c, d$ and $f$ were generated using the PanCancer Atlas (all copy number data in Fig. $6 \mathrm{c}$ and d; ESCA, LUSC, UCEC, BRCA data in Fig. 6f) and METABRIC datasets (in Fig. 6f) in CBioPortal ${ }^{81,82}$ (https://www.cbioportal.org/). Source data are provided with this paper.

Received: 8 April 2020; Accepted: 2 November 2021; Published online: 29 November 2021

\section{References}

1. Neganova, I. \& Lako, M. G1 to S phase cell cycle transition in somatic and embryonic stem cells. J. Anat. 213, 30-44 (2008).

2. Khoronenkova, S. V. \& Dianov, G. L. ATM prevents DSB formation by coordinating SSB repair and cell cycle progression. Proc. Natl Acad. Sci. USA 112, 3997-4002 (2015)

3. Macheret, M. \& Halazonetis, T. D. Intragenic origins due to short G1 phases underlie oncogene-induced DNA replication stress. Nature 555, 112-116 (2018).

4. Halazonetis, T. D., Gorgoulis, V. G. \& Bartek, J. An oncogene-induced DNA damage model for cancer development. Science 319, 1352-1355 (2008).

5. Hills, S. A. \& Diffley, J. F. X. DNA replication and oncogene-induced replicative stress. Curr. Biol. 24, R435-R444 (2014).

6. Hume, S., Dianov, G. L. \& Ramadan, K. A unified model for the G1/S cell cycle transition. Nucleic Acids Res. 48, 12483-12501 (2020).

7. Pennycook, B. R. \& Barr, A. R. Restriction point regulation at the crossroads between quiescence and cell proliferation. FEBS Lett. https://doi.org/10.1002/ 1873-3468.13867 (2020).

8. Rubin, S. M., Sage, J. \& Skotheim, J. M. Integrating old and new paradigms of G1/S control. Mol. Cell 80, 183-192 (2020).

9. Bartek, J. \& Lukas, J. Pathways governing G1/S transition and their response to DNA damage. FEBS Lett. 490, 117-122 (2001).

10. Spencer, S. L. et al. The proliferation-quiescence decision is controlled by a bifurcation in CDK2 activity at mitotic exit. Cell 155, 369-383 (2013).

11. Yang, H. W., Chung, M., Kudo, T. \& Meyer, T. Competing memories of mitogen and p53 signalling control cell-cycle entry. Nature https://doi.org/ 10.1038/nature23880 (2017).

12. Heldt, F. S., Barr, A. R., Cooper, S., Bakal, C. \& Novak, B. A comprehensive model for the proliferation-quiescence decision in response to endogenous DNA damage in human cells. Proc. Natl Acad. Sci. USA 115, 2532-2537 (2018).

13. Barr, A. R. et al. DNA damage during S-phase mediates the proliferationquiescence decision in the subsequent G1 via p21 expression. Nat. Commun. 8, 14728 (2017).

14. Barr, A. R., Heldt, F. S., Zhang, T., Bakal, C. \& Novák, B. A dynamical framework for the all-or-none G1/S transition. Cell Syst. 2, 27-37 (2016).

15. Bianchi, M. E. \& Agresti, A. HMG proteins: dynamic players in gene regulation and differentiation. Curr. Opin. Genet. Dev. 15, 496-506 (2005).

16. Qiu, B. et al. NUCKS is a positive transcriptional regulator of insulin signaling. Cell Rep. 7, 1876-1886 (2014).

17. Yuan, X. et al. NUCKS1 is a novel regulator of milk synthesis in and proliferation of mammary epithelial cells via the mTOR signaling pathway. $J$. Cell. Physiol. https://doi.org/10.1002/jcp.28240 (2019).

18. Huang, Y.-K. et al. NUCKS1 promotes gastric cancer cell aggressiveness by upregulating IGF-1R and subsequently activating the PI3K/Akt/mTOR signaling pathway. Carcinogenesis 40, 370-379 (2019).
19. Parplys, A. C. et al. NUCKS1 is a novel RAD51AP1 paralog important for homologous recombination and genome stability. Nucleic Acids Res. 43, 9817-9834 (2015)

20. Yue, Y. et al. Nucks1 synergizes with Trp53 to promote radiation lymphomagenesis in mice. Oncotarget 7, 61874-61889 (2016).

21. De Angelis, P. M. et al. Nondysplastic ulcerative colitis has high levels of the homologous recombination repair protein NUCKS1 and low levels of the DNA damage marker gamma-H2AX. Inflamm. Bowel Dis. 24, 593-600 (2018).

22. Maranon, D. G. et al. NUCKS1 promotes RAD54 activity in homologous recombination DNA repair. J. Cell Biol. 219, e201911049 (2020).

23. Wisniewski, J. R. et al. Constitutive and dynamic phosphorylation and acetylation sites on NUCKS, a hypermodified nuclear protein, studied by quantitative proteomics. Proteins 73, 710-718 (2008).

24. Malumbres, M. \& Barbacid, M. Cell cycle, CDKs and cancer: a changing paradigm. Nat. Rev. Cancer 9, 153-166 (2009).

25. Ostvold, A. C., Holtlund, J. \& Laland, S. G. A novel, highly phosphorylated protein, of the high-mobility group type, present in a variety of proliferating and non-proliferating mammalian cells. Eur. J. Biochem. 153, 469-475 (1985).

26. Grundt, K. et al. A putative DNA-binding domain in the NUCKS protein. Arch. Biochem. Biophys. 407, 168-175 (2002).

27. Grundt, K., Haga, I. V., Huitfeldt, H. S. \& Ostvold, A. C. Identification and characterization of two putative nuclear localization signals (NLS) in the DNA-binding protein NUCKS. Biochim. Biophys. Acta 1773, 1398-1406 (2007).

28. Ostvold, A. C. et al. Molecular cloning of a mammalian nuclear phosphoprotein NUCKS, which serves as a substrate for Cdk1 in vivo. Eur. J. Biochem. 268, 2430-2440 (2001).

29. Matsuoka, S. et al. ATM and ATR substrate analysis reveals extensive protein networks responsive to DNA damage. Science 316, 1160-1166 (2007).

30. Grundt, K., Thiede, B. \& Østvold, A. C. Identification of kinases phosphorylating 13 sites in the nuclear, DNA-binding protein NUCKS. Biochim. Biophys. Acta Proteins Proteom. 1865, 359-369 (2017).

31. Chicas, A. et al. Dissecting the unique role of the retinoblastoma tumor suppressor during cellular senescence. Cancer Cell 17, 376-387 (2010).

32. Nikulenkov, F. et al. Insights into p53 transcriptional function via genomewide chromatin occupancy and gene expression analysis. Cell Death Differ. 19, 1992-2002 (2012)

33. Qiu, B., Han, W. \& Tergaonkar, V. NUCKS: a potential biomarker in cancer and metabolic disease. Clin. Sci. 128, 715-721 (2015).

34. Shi, C. et al. NUCKS nuclear elevated expression indicates progression and prognosis of ovarian cancer. Tumour Biol. 39, 1010428317714631 (2017).

35. Liu, T. et al. Increased NUCKS expression is a risk factor for poor prognosis and recurrence in endometrial cancer. Am. J. Cancer Res. 5, 3659-3667 (2015).

36. Drosos, Y. et al. NUCKS overexpression in breast cancer. Cancer Cell Int. 9, 19 (2009).

37. Kikuchi, A. et al. Identification of NUCKS1 as a colorectal cancer prognostic marker through integrated expression and copy number analysis. Int. J. Cancer 132, 2295-2302 (2013).

38. Gu, L. et al. NUCKS1 overexpression is a novel biomarker for recurrence-free survival in cervical squamous cell carcinoma. Tumour Biol. 35, 7831-7836 (2014).

39. Cheong, J. Y. et al. Identification of NUCKS1 as a putative oncogene and immunodiagnostic marker of hepatocellular carcinoma. Gene 584, 47-53 (2016).

40. Zhao, S. et al. NUCKS1 promotes proliferation, invasion and migration of non-small cell lung cancer by upregulating CDK1 expression. Cancer Manag. Res. 12, 13311-13323 (2020).

41. Cardozo, T. \& Pagano, M. The SCF ubiquitin ligase: insights into a molecular machine. Nat. Rev. Mol. Cell Biol. 5, 739-751 (2004).

42. Abbas, T. \& Dutta, A. p21 in cancer: intricate networks and multiple activities Nat. Rev. Cancer 9, 400-414 (2009).

43. Chu, I. M., Hengst, L. \& Slingerland, J. M. The Cdk inhibitor p27 in human cancer: prognostic potential and relevance to anticancer therapy. Nat. Rev. Cancer 8, 253-267 (2008).

44. Carrano, A. C., Eytan, E., Hershko, A. \& Pagano, M. SKP2 is required for ubiquitin-mediated degradation of the CDK inhibitor p27. Nat. Cell Biol. 1, 193-199 (1999).

45. Bornstein, G. et al. Role of the SCFSkp2ubiquitin ligase in the degradation of p21Ciplin S phase. J. Biol. Chem. https://doi.org/10.1074/jbc.M301774200 (2003).

46. Frescas, D. \& Pagano, M. Deregulated proteolysis by the F-box proteins SKP2 and beta-TrCP: tipping the scales of cancer. Nat. Rev. Cancer 8, 438-449 (2008).

47. Zhu, Q. et al. Targeted exploration and analysis of large cross-platform human transcriptomic compendia. Nat. Methods 12, 211-214 (2015). 3 p following 214.

48. Kitagawa, M., Lee, S. H. \& McCormick, F. Skp2 suppresses p53-dependent apoptosis by inhibiting p300. Mol. Cell 29, 217-231 (2008). 
49. Wu, J. et al. Skp2 E3 ligase integrates ATM activation and homologous recombination repair by ubiquitinating NBS1. Mol. Cell 46, 351-361 (2012).

50. Imaki, H. et al. Cell cycle-dependent regulation of the Skp2 promoter by GAbinding protein. Cancer Res. 63, 4607-4613 (2003).

51. Yam, C. H., Fung, T. K. \& Poon, R. Y. C. Cyclin A in cell cycle control and cancer. Cell. Mol. Life Sci. 59, 1317-1326 (2002).

52. Bashir, T., Dorrello, N. V., Amador, V., Guardavaccaro, D. \& Pagano, M. Control of the SCF(Skp2-Cks1) ubiquitin ligase by the APC/C(Cdh1) ubiquitin ligase. Nature 428, 190-193 (2004).

53. Moldovan, G.-L., Pfander, B. \& Jentsch, S. PCNA, the maestro of the replication fork. Cell 129, 665-679 (2007).

54. Schroering, A. G., Edelbrock, M. A., Richards, T. J. \& Williams, K. J. The cell cycle and DNA mismatch repair. Exp. Cell Res. 313, 292-304 (2007).

55. Meloche, S. \& Pouysségur, J. The ERK1/2 mitogen-activated protein kinase pathway as a master regulator of the G1- to S-phase transition. Oncogene 26, 3227-3239 (2007).

56. Vousden, K. H. \& Prives, C. Blinded by the light: the growing complexity of p53. Cell 137, 413-431 (2009).

57. Engeland, K. Cell cycle arrest through indirect transcriptional repression by p53: I have a DREAM. Cell Death Differ. 25, 114-132 (2018).

58. Fischer, M., Quaas, M., Steiner, L. \& Engeland, K. The p53-p21-DREAM CDE/CHR pathway regulates G2/M cell cycle genes. Nucleic Acids Res. 44, 164-174 (2016).

59. Poletto, M., Legrand, A. J., Fletcher, S. C. \& Dianov, G. L. p53 coordinates base excision repair to prevent genomic instability. Nucleic Acids Res. 44, 3165-3175 (2016).

60. Yokoi, S. et al. Amplification and overexpression of SKP2 are associated with metastasis of non-small-cell lung cancers to lymph nodes. Am. J. Pathol. 165, 175-180 (2004).

61. Zhu, C. Q. et al. Skp2 gene copy number aberrations are common in nonsmall cell lung carcinoma, and its overexpression in tumors with ras mutation is a poor prognostic marker. Clin. Cancer Res. 10, 1984-1991 (2004).

62. Gstaiger, M. et al. Skp2 is oncogenic and overexpressed in human cancers. Proc. Natl Acad. Sci. USA 98, 5043-5048 (2001).

63. Rose, A. E. et al. Clinical relevance of SKP2 alterations in metastatic melanoma. Pigment Cell Melanoma Res. 24, 197-206 (2011).

64. Muller, P. A. J. \& Vousden, K. H. p53 mutations in cancer. Nat. Cell Biol. 15, 2-8 (2013).

65. Poon, M.-W. et al. Inhibition of NUCKS facilitates corneal recovery following alkali burn. Sci. Rep. 7, 41224 (2017).

66. Schneider, G. et al. IKKalpha controls $\mathrm{p} 52 / \mathrm{RelB}$ at the skp2 gene promoter to regulate G1- to S-phase progression. EMBO J. 25, 3801-3812 (2006)

67. Bensimon, A. et al. ATM-dependent and -independent dynamics of the nuclear phosphoproteome after DNA damage. Sci. Signal. 3, rs3 (2010).

68. Ren, B. et al. E2F integrates cell cycle progression with DNA repair, replication, and G(2)/M checkpoints. Genes Dev. 16, 245-256 (2002).

69. Stead, L. F. et al. The transcriptional consequences of somatic amplifications, deletions, and rearrangements in a human lung squamous cell carcinoma. Neoplasia 14, 1075-1086 (2012).

70. McKinley, K. L. \& Cheeseman, I. M. Large-scale analysis of CRISPR/Cas9 cellcycle knockouts reveals the diversity of p53-dependent responses to cell-cycle defects. Dev. Cell 40, 405-420.e2 (2017).

71. Tajima, K. et al. SETD1A protects from senescence through regulation of the mitotic gene expression program. Nat. Commun. 10, 2854 (2019).

72. Bunz, F. et al. Requirement for p53 and p21 to sustain G2 arrest after DNA damage. Science https://doi.org/10.1126/science.282.5393.1497 (1998).

73. Orlando, G., Khoronenkova, S. V., Dianova, I. I., Parsons, J. L. \& Dianov, G. L. ARF induction in response to DNA strand breaks is regulated by PARP1. Nucleic Acids Res. 42, 2320-2329 (2014).

74. Halder, S. et al. SPRTN protease and checkpoint kinase 1 cross-activation loop safeguards DNA replication. Nat. Commun. 10, 3142 (2019).

75. Bushell, S. R. et al. The structural basis of lipid scrambling and inactivation in the endoplasmic reticulum scramblase TMEM16K. Nat. Commun. 10, 3956 (2019).

76. Carpenter, A. E. et al. CellProfiler: image analysis software for identifying and quantifying cell phenotypes. Genome Biol. 7, R100 (2006).

77. Burdova, K. et al. E2F1 proteolysis via SCF-cyclin F underlies synthetic lethality between cyclin F loss and Chk1 inhibition. EMBO J. e101443 https:// doi.org/10.15252/embj.2018101443 (2019).

78. Tabas-Madrid, D., Nogales-Cadenas, R. \& Pascual-Montano, A. GeneCodis3: a non-redundant and modular enrichment analysis tool for functional genomics. Nucleic Acids Res. 40, W478-W483 (2012).
79. Tang, Z., Kang, B., Li, C., Chen, T. \& Zhang, Z. GEPIA2: an enhanced web server for large-scale expression profiling and interactive analysis. Nucleic Acids Res. https://doi.org/10.1093/nar/gkz430 (2019).

80. Goldman, M. J. et al. Visualizing and interpreting cancer genomics data via the Xena platform. Nat. Biotechnol. 38, 675-678 (2020).

81. Cerami, E. et al. The cBio cancer genomics portal: an open platform for exploring multidimensional cancer genomics data. Cancer Discov. 2, 401-404 (2012).

82. Gao, J. et al. Integrative analysis of complex cancer genomics and clinical profiles using the cBioPortal. Sci. Signal. 6, pl1 (2013).

\section{Acknowledgements}

The authors thank Prof. I. Cheeseman (Department of Biology, Massachusetts Institute of Technology), Dr. R. Chapman (Nuffield Department of Medicine, University of Oxford) and Prof. S. Maheswaran (Massachusetts General Hospital Cancer Center, Harvard Medical School) for providing cell lines. We thank Prof. E. O'Neill (Departmen of Oncology, University of Oxford) for providing plasmids, Prof. A. Østvold (Department of Biochemistry, University of Oslo) for NUCKS1 antibodies, and Dr. S. Mukhopadhyay, Prof. N. Burgess-Brown (Structural Genomics Consortium, University of Oxford), and Dr. S. Khoronenkova (Department of Biochemistry, University of Cambridge) for their help with NUCKS1 purification. The authors thank present and past members of the Dianov and Ramadan labs for discussions and technical help. G.L.D. is supported by grants from the Medical Research Council [H3RWGJ00.H302.1], Cancer Research UK [C5255/A15935], and the Russian Science Foundation grant (№19-7420069). K.R. is supported by the Medical Research Council Programme (MC_PC-12001/ 1 and MC_UU-00001/1) and Breast Cancer Now (2019DecPR1406). S.H. was supported by the Radcliffe-Oncology Studentship at University College, University of Oxford.

\section{Author contributions}

G.L.D. and A.J.L. conceived the study and were in charge of overall direction and planning. S.H., C.P.G., P.L. and A.J.L. performed experiments. All authors designed and analysed experiments. V.D. gave critical suggestions and provided reagents. A.J.L., K.R and G.L.D. supervised the project. S.H., A.J.L., K.R. and G.L.D. wrote the manuscript. All authors read and approved the manuscript.

\section{Competing interests}

The authors declare no competing interests.

\section{Additional information}

Supplementary information The online version contains supplementary material available at https://doi.org/10.1038/s41467-021-27124-8.

Correspondence and requests for materials should be addressed to Arnaud J. Legrand, Kristijan Ramadan or Grigory L. Dianov.

Peer review information Nature Communications thanks Ian Cannell, and the other, anonymous, reviewer(s) for their contribution to the peer review of this work.

Reprints and permission information is available at http://www.nature.com/reprints

Publisher's note Springer Nature remains neutral with regard to jurisdictional claims in published maps and institutional affiliations.

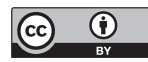

Open Access This article is licensed under a Creative Commons Attribution 4.0 International License, which permits use, sharing, adaptation, distribution and reproduction in any medium or format, as long as you give appropriate credit to the original author(s) and the source, provide a link to the Creative Commons license, and indicate if changes were made. The images or other third party material in this article are included in the article's Creative Commons license, unless indicated otherwise in a credit line to the material. If material is not included in the article's Creative Commons license and your intended use is not permitted by statutory regulation or exceeds the permitted use, you will need to obtain permission directly from the copyright holder. To view a copy of this license, visit http://creativecommons.org/ licenses/by/4.0/.

(c) The Author(s) 2021 